\title{
CHROMATIC EXPANSIONS IN FUNCTION SPACES
}

\author{
AHMED I. ZAYED
}

\begin{abstract}
Chromatic series expansions of bandlimited functions have recently been introduced in signal processing with promising results. Chromatic series share similar properties with Taylor series insofar as the coefficients of the expansions, which are called chromatic derivatives, are based on the ordinary derivatives of the function, but unlike Taylor series, chromatic series have a better rate of convergence and more practical applications.

The $n$-th chromatic derivative $K^{n}(f)$ of an analytic function $f(t)$ is a linear combination of the ordinary derivatives $f^{(k)}(t), 0 \leq k \leq n$, where the coefficients of the combination are based on systems of orthogonal polynomials. In addition to their practical applications, chromatic series expansions have useful theoretical and mathematical applications. For example, functions in the Paley-Wiener space can be completely characterized by their chromatic series expansions associated with the Legendre polynomials.

The purpose of this paper is to show that chromatic series expansions can be used to characterize other important function spaces. We show that functions in weighted Bergman spaces $\mathfrak{B}_{\gamma}$ can be characterized by their chromatic series expansions that use chromatic derivatives associated with the Laguerre polynomials, while functions in the Bargmann-Segal-Foch space $\mathfrak{F}$ can be characterized by their chromatic series expansions that use chromatic derivatives associated with the Hermite polynomials. Another goal of this article is to show that each one of these spaces has an orthonormal basis that is generated from one single function $\psi$ by applying successive chromatic derivatives to it, that is, both $\mathfrak{B}_{\gamma}$ and $\mathfrak{F}$ have an orthonormal basis of the form $\left\{K^{n} \psi\right\}_{n=0}^{\infty}$.
\end{abstract}

\section{INTRODUCTION}

Chromatic derivatives and series expansions have recently been introduced in signal analysis by A. Ignjatovic in [18,19] as an alternative representation to Taylor series for bandlimited functions, and they have been shown to be more useful in practical applications than Taylor series; see [4, 6, 2, 15, 17, 29, 30, 34, 35]. For some related ideas but in a different context see [25, 26].

Recall that a function $f$ is bandlimited to $[-\sigma, \sigma]$ if it can be represented as

$$
f(t)=\int_{-\sigma}^{\sigma} e^{-i x t} g(x) d x, \quad t \in \mathbb{R},
$$

for some function $g \in L^{2}(-\sigma, \sigma)$.

The space of bandlimited functions, which is also known as the Paley-Wiener space of bandlimited functions, consists of entire functions of exponential type that are square integrable on the real axis. The Paley-Wiener space of functions bandlimited to $[-\sigma, \sigma]$ will be denoted by $P W_{\sigma}$. The Whittaker-Shannon-Kotel'nikov

Received by the editors August 17, 2011 and, in revised form, July 20, 2012.

2010 Mathematics Subject Classification. Primary 41A58, 42C15; Secondary 44A15, 42B35.

Key words and phrases. Chromatic derivatives, chromatic series expansions, weighted Bergman spaces, the Bargmann-Segal-Foch space, the Bargmann transform.

(C) 2014 American Mathematical Society 4097

Reverts to public domain 28 years from publication 
(WSK) sampling theorem states that if $f \in P W_{\sigma}$, then it can be reconstructed from its samples, $f(k \pi / \sigma)$. The construction formula is

$$
f(t)=\sum_{k=-\infty}^{\infty} f\left(\frac{k \pi}{\sigma}\right) \frac{\sin (\sigma t-k \pi)}{(\sigma t-k \pi)}, \quad t \in \mathbb{R},
$$

with the series being absolutely and uniformly convergent on compact subsets of $\mathbb{R}$. See, e.g., [38, p. 16].

The WSK theorem plays an important role in communication engineering because it enables engineers to reconstruct an analogue signal from its samples at a discrete set of points. The WSK expansion may be viewed as a global expansion because it uses function values at infinitely many points uniformly distributed on the real line.

On the other hand, as an entire function, $f$ has a Taylor series expansion of the form $f(t)=\sum_{n=0}^{\infty}\left(f^{(n)}(0) / n !\right) t^{n}$, which may be viewed as a local expansion since it uses the values of $f$ and all its derivatives at a single point.

While the Whittaker-Shannon-Kotel'nikov sampling expansion has played an important role in digital signal processing applications, the Taylor series has limited practical applications. This can be attributed to two facts.

- The Sinc function, Sinc $x=\sin \pi x / \pi x$, belongs to $P W_{\pi}$, and its translates, $\{\operatorname{sinc}(t-n)\}_{n \in \mathbb{Z}}$, form an orthogonal basis for $P W_{\pi}$. Hence any $f \in P W_{\pi}$ can be approximated by truncating its sampling series, while a truncated Taylor series, i.e., a polynomial of degree $n$, is not bandlimited.

- Function evaluation is easier to compute and more numerically stable than numerical evaluation of derivatives.

Another representation of bandlimited functions is provided by the chromatic series expansions which are based on the notion of chromatic derivatives.

The $n$-th chromatic derivative $K^{n}[f]\left(t_{0}\right)$ of an analytic function $f(t)$, at $t_{0}$, whose formal definition will be given in the next section, is a linear combination of the ordinary derivatives $f^{(k)}\left(t_{0}\right), 0 \leq k \leq n$, where the coefficients of the combination are based on systems of orthogonal polynomials. However, unlike the ordinary derivatives, the chromatic derivatives can be obtained more accurately in a noise robust way. Although there are several notions of derivatives available in the literature, such as symmetric, Peano, $L^{p}$, symmetric $L^{p}$, quantum, Fréchet, and Gateaux derivatives, they are all mathematical generalizations of the ordinary derivative and are used to describe the local behavior of a function in a neighborhood of the point of differentiation. In contrast, chromatic derivatives arose from real world applications, and analogous to ordinary derivatives they can be used to describe the global behavior of analytic functions.

Although chromatic series are like Taylor series, locally in nature, they provide more numerically robust expansions than their Taylor counterparts. The transfer functions of ordinary derivatives cluster tightly together and obliterate all but the edges of the spectrum of a bandlimited signal. On the contrary, the transfer functions of chromatic derivatives form a family of well-separated, interleaved refined comb filters; see [4,30,33.

The chromatic series expansion of $f \in C^{\infty}(\mathbb{R})$ is given by the series

$$
f(z) \sim \sum_{n=0}^{\infty} K^{n}(f)(0) K^{n}(\psi)(z),
$$


for some appropriate function $\psi$ that depends on the associated system of orthogonal polynomials used. To demonstrate the idea, let $\left\{P_{n}(\omega)\right\}_{n=0}^{\infty}$ be a family of polynomials orthonormal with respect to a weight function $W(\omega)$ having finite moments and satisfying some growth conditions with

$$
\int_{-\infty}^{\infty} e^{c|\omega|} W(\omega) d \omega<\infty
$$

for some $c>0$, so that

$$
\int_{-\infty}^{\infty} P_{n}(\omega) P_{m}(\omega) W(\omega) d \omega=\delta_{m, n}
$$

Then for any $f(z)$ analytic in the strip $S(c / 2)=\{z: \Im(z)<c / 2\}$ and for which the series $\sum_{n=0}^{\infty}\left|K^{n}(f)(0)\right|^{2}$ converges, the series (1.3) converges to $f(z)$ uniformly in every strip $\{z:|\Im(z)|<c / 2-\varepsilon\}$, for any $\varepsilon>0$, where

$$
\psi(z)=\int_{-\infty}^{\infty} e^{i \omega z} W(\omega) d \omega .
$$

It is easy to see that $\psi(z)$ is analytic in the strip $S(c / 2)=\{z: \Im(z)<c / 2\}$.

In the particular case where $W(\omega)=\chi_{(-\pi, \pi)}$, and $\left\{P_{n}(\omega)\right\}_{n=0}^{\infty}$ are the Legendre polynomials normalized such that

$$
\int_{-\pi}^{\pi} P_{n}(\omega) P_{m}(\omega) W(\omega) d \omega=2 \pi \delta_{m, n}
$$

then the series (1.3) takes the form

$$
f(z)=\sum_{n=0}^{\infty} K^{n}(f)(0) K^{n}(\psi)(z),
$$

for any bandlimited function $f \in P W_{\pi}$, where $\psi(z)=\operatorname{Sinc} z$ and in this case the strip $S(c / 2)$ is the entire plane.

Chromatic derivatives are intrinsically related to the Fourier transformation. They are constructed using the fact that, under the Fourier transformation, differentiation in the time domain corresponds to multiplication by powers of $\omega$ in the frequency domain.

In two recent papers 36, 37 we introduced more general types of chromatic derivatives and series that are better suited to handle more general integral transforms than the Fourier transform. In 37] we presented two different methods to construct a differential operator $L$ that gives rise to generalized chromatics derivatives and their associated integral transform. In the first method the operator $L$ arises from certain Sturm-Liouville boundary-value problems, while in the second, it arises from initial-value problems involving differential operators of order $n$.

It was shown that in the first method the functions $\left\{K^{n} \psi(z)\right\}$ form an orthonormal basis of a certain function space, while in the second method they may form a Riesz basis. That is, the basis may be generated from one single function by applying successive chromatic derivatives to it. This is reminiscent of the wavelets and Gabor systems in which orthonormal bases of certain function spaces are generated from one single function by translation and dilation in the former case and by translation and modulation in the latter. More specifically, if we denote the translation, dilation, and modulation operators respectively by $T_{m}, D^{n}, E_{k}$ where

$$
T_{m} f(x)=f(x-m), \quad D^{n} f(x)=2^{n / 2} f\left(2^{n} x\right), \quad E_{k} f(x)=e^{i\langle k, x\rangle} f(x),
$$


where $x \in \mathbb{R}^{d}, m, k \in \mathbb{Z}^{d}, n \in \mathbb{Z}$, then the wavelet and Gabor systems on $\mathbb{R}^{d}$ can be written, respectively, as

$$
\psi_{m, n}(x)=D^{n} T_{m} \psi(x), \quad g_{m, k}(x)=E_{k} T_{m} g(x) .
$$

Of special importance are the cases in which the wavelets or the Gabor systems form orthonormal, or Riesz bases or frames for some function spaces. Analogously, we shall show that there exists an entire function $\psi$ such that $\left\{K^{\alpha} \psi(z)\right\}_{\alpha}$ is an orthonormal basis for the Bargmann-Segal-Foch space $\mathfrak{F}$, where $\alpha=\left(\alpha_{1}, \cdots, \alpha_{d}\right)$ is a multi-index and $\alpha_{i} \in \mathbb{N}$.

The purpose of this article is to show that chromatic series can be used like Taylor series to characterize spaces of analytic functions. This work extends Ignjatovic's result for bandlimited functions; see (1.4). In fact, in some cases the chromatic expansion of a function $f$ is just an orthogonal expansion in terms of an orthogonal basis generated by one single function. We will demonstrate this idea for two important spaces of analytic functions: a generalized Bergman space of analytic functions in the unit disk $\mathfrak{B}_{\gamma}$ and the Bargmann-Segal-Foch space $\mathfrak{F}$ of entire functions in $\mathbb{C}^{n}$. We shall utilize the Bargmann transform and chromatic derivatives associated with the Hermite polynomials to obtain an orthonormal basis for the space $\mathfrak{F}$.

The notation we use is standard. We denote the set of real numbers by $\mathbb{R}$, the integers by $\mathbb{Z}$, and the natural numbers by $\mathbb{N}$.

The article is organized as follows. In Section 2 we give a brief introduction to chromatic derivatives and series. A more general form of chromatic series, which will be used to derive the main results of the article, is presented in Section 3 , In Section 4 we derive the chromatic expansions in a generalized Bergman space of analytic functions in the unit disk. Because chromatic derivatives in higher dimensions are based on orthogonal polynomials in several variables, we will give a brief introduction to the theory of orthogonal polynomials in several variables in Section 5. followed by an introduction to chromatic derivatives and series in higher dimensions in Section 6. Sections 7 and 8 give a brief introduction to the BargmannSegal-Foch space and the Bargmann transform and some of their properties that will be used in the sequel. The main results are presented in Section9. We conclude the article in Section 10 with some remarks and applications to asymptotic expansions and solving differential equations.

\section{Chromatic Derivatives And Series}

In this section we describe the general idea of chromatic expansions associated with a family of orthogonal polynomials.

For the reader's convenience, we will briefly describe how chromatic series are constructed in one dimension and relegate the treatment of higher dimensions to Section 6. Let $W(\omega)$ be a nonnegative weight function such that all of its moments are finite, i.e., such that

$$
\mu_{n}=\int_{-\infty}^{\infty} \omega^{n} W(\omega) d \omega<\infty
$$

Let $\left\{P_{n}(\omega)\right\}_{n=0}^{\infty}$ be the family of polynomials orthonormal with respect to $W(\omega)$ :

$$
\int_{-\infty}^{\infty} P_{n}(\omega) P_{m}(\omega) W(\omega) d \omega=\delta_{m, n}
$$


and let $K^{n}(f)=P_{n}\left(i \frac{d}{d t}\right)(f)$ be the corresponding linear differential operator obtained from $P_{n}(\omega)$ by replacing $\omega^{k}(0 \leq k \leq n)$ with $i^{k} \frac{d^{k}}{d t^{k}}$. These differential operators are called chromatic derivatives associated with the family of orthogonal polynomials $\left\{P_{n}(\omega)\right\}$ because they preserve the spectral features of bandlimited signals. They can be evaluated with high accuracy and in a noise robust way from samples of the signal taken at a small multiple of the usual Nyquist rate; see [13, 15, 16] for details.

Let $\psi(z)$ be the Fourier transform of the weight function $W(\omega)$,

$$
\psi(z)=\int_{-\infty}^{\infty} e^{i \omega z} W(\omega) d \omega
$$

Because $\psi(z)$ will be used in a Taylor-type expansion of functions analytic in a domain around the origin, we shall assume that $\lim \sup \left(\mu_{n} / n !\right)^{1 / n}<\infty$, where, $\psi^{(n)}(0)=i^{n} \mu_{n}$. This condition implies that $\psi(z)$ is analytic around the origin. As shown in [14, this condition holds if and only if

$$
\int_{-\infty}^{\infty} e^{c|\omega|} W(\omega) d \omega<\infty
$$

for some $c>0$, and in this case $\psi(z)$ is analytic in the strip $S(c / 2)=\{z: \Im(z)<$ $c / 2\}$.

The chromatic series expansion of $f \in C^{\infty}(\mathbb{R})$ is given by the following formal series:

$$
f(z) \sim \sum_{n=0}^{\infty} K^{n}(f)(0) K^{n}(\psi)(z) .
$$

It has been shown in [14] that if $f(z)$ is analytic in the strip $S(c / 2)$ and $\sum_{n=0}^{\infty}\left|K^{n}(f)(0)\right|^{2}$ converges, then the series (2.1) converges to $f(z)$, uniformly in every strip $\{z:|\Im(z)|<c / 2-\varepsilon\}$, for any $\varepsilon>0$. Here it should be emphasized that although the chromatic series were originally introduced for bandlimited functions, the theory now applies to a much larger class of functions.

In the particular case, where $W(\omega)=\chi_{(-1,1)}$, the chromatic series associated with the Legendre polynomials converge in the whole complex plane, i.e., the strip $S(c / 2)$ is $\mathbb{C}$, and the set of entire functions for which $\sum_{n=0}^{\infty}\left|K^{n}(f)(0)\right|^{2}$ converges is precisely the set of $L^{2}$ functions whose Fourier transforms are finitely supported, i.e., the set of bandlimited functions. For such functions the chromatic expansions converge uniformly on $\mathbb{R}$, and their truncated series are themselves bandlimited, which is analogous to the Whittaker-Shannon sampling series [38. This is in contrast to Taylor series whose truncated series are not bandlimited. For this reason chromatic series have more practical applications in signal processing than Taylor series.

\section{Chromatic Derivatives associated}

\section{WITH MORE GENERAL DIFFERENTIAL OPERATORS}

In this section we summarize two generalizations of chromatic derivatives and their associated chromatic series. 
3.1. Chromatic derivatives associated with a Sturm-Liouville differential operator. Consider the singular Sturm-Liouville boundary-value problem on the half-line,

$$
\begin{gathered}
L y=-y^{\prime \prime}+q(x) y=\lambda y, \quad 0 \leq x<\infty, \\
y(0) \cos \alpha+y^{\prime}(0) \sin \alpha=0, \quad-\pi<\alpha \leq \pi,
\end{gathered}
$$

where $q(x) \in L^{1}\left(\mathbb{R}^{+}\right)$is real valued. It is known that the condition $q \in L^{1}\left(\mathbb{R}^{+}\right)$ implies that the problem is in the limit point case at $\infty$ and that the spectrum is continuous [32]. In fact, there exists a nondecreasing function $\rho(\lambda)$ such that for all $f \in L^{2}\left(\mathbb{R}^{+}\right)$,

$$
\hat{f}(\lambda)=\int_{0}^{\infty} f(x) \phi(x, \lambda) d x
$$

exists in the mean and defines a function $\hat{f}(\lambda)$ such that

$$
f(x)=\int_{-\infty}^{\infty} \hat{f}(\lambda) \phi(x, \lambda) d \rho(\lambda)
$$

where $\phi(x, \lambda)$ is a solution of the differential equation (3.1) that satisfies the initial condition

$$
\phi(0, \lambda)=\sin \alpha, \phi^{\prime}(0, \lambda)=-\cos \alpha .
$$

We call the integral transform (3.3) the $\phi$-transform of $f$. Fix $0<a<\infty$, and let $K^{2}(a)$ denote the set of all functions with supports in $[0, a]$ that are square integrable with respect to $d \rho$. In most cases of interest $d \rho$ is supported on a half-line which, without loss of generality, we may take as $[0, \infty)$. For sufficient conditions for this to hold see [27, p. 128]. The main result can be summarized in the following theorem whose proof can be found in [37].

Theorem 3.1. Consider the boundary-value problem (3.1) and (3.2) and let $C K^{2}(a)$ denote the image of $K^{2}(a)$ under the transformation (3.4). Then there exists a sequence of polynomials $\left\{p_{n}(\lambda)\right\}_{n=0}^{\infty}$ that are orthonormal with respect to $d \rho$ on $[0, a]$ and $p_{n}(\lambda)$ is of exact degree $n$. Furthermore, for any $f \in C K^{2}(a)$ we have for $\alpha \neq 0, \pi$

$$
f(x)=\frac{1}{(\sin \alpha)} \sum_{n=0}^{\infty}\left[p_{n}(L) f\right](0) \psi_{n}(x),
$$

where

$$
\psi_{n}(x)=\int_{0}^{\infty} p_{n}(\lambda) \phi(x, \lambda) d \rho(\lambda)
$$

and the series converges to $f$ in the mean. Similar expressions exist for $\alpha=0$ or $\pi$. The functions $\left\{\psi_{n}(x)\right\}$ are orthonormal on $[0, \infty)$ and satisfy the initial condition (3.5). The series (3.6) converges to $f(x)$ pointwise for $0 \leq x<\infty$. In fact, the series converges to $f$ uniformly on compact subsets of $(0, \infty)$.

Definition 3.2. The $n$-th generalized chromatic derivative of a function $f$ associated with the differential operator $L$ at $x=0$ is defined as

$$
K^{n}[f](0)=\left\langle\hat{f}, p_{n}\right\rangle_{d \rho}=\frac{1}{(\sin \alpha)}\left[p_{n}(L) f\right](0), \text { for } \alpha \neq 0, \pi,
$$


where $\hat{f}$ is the $\phi$ transform of $f$. Analogous to (2.1), we define the generalized chromatic series expansion of $f$ as

$$
\sum_{n=0}^{\infty} K^{n}[f](0) K^{n}[\psi](x), \text { where } \psi_{n}(x)=K^{n}[\psi](x),
$$

and

$$
\psi(x)=\int_{0}^{a} \phi(x, \lambda) d \rho(\lambda) .
$$

3.2. Chromatic series associated with more general integral transforms. In this section we briefly introduce another generalization of chromatic series that is more intrinsically related to more general integral transforms than the Fourier transform. We begin with an integral transform and assume that the kernel of the transform arises from an initial-value problem associated with a linear differential operator with variable coefficients. It should be noted that the kernels of most classical integral transforms, such as the Fourier, Laplace, Hankel, and Legendre transforms, possess this property.

Consider the integral transform

$$
\int_{J_{1}} \hat{f}(\lambda) \phi(x, \lambda) d \lambda
$$

where $J_{1}$ is either $[0, \infty)$ or $(-\infty, \infty)$. Assume that the kernel function $\phi(x, \lambda)$ satisfies the differential equation

$$
L \phi(x, \lambda)=\lambda \phi(x, \lambda), \quad x \in I,
$$

on some interval $I$, where

$$
L=q_{n}(x) \frac{d^{n}}{d x^{n}}+q_{n-1}(x) \frac{d^{n-1}}{d x^{n-1}}+\ldots+q_{1}(x) \frac{d}{d x}+q_{0}(x)
$$

for some continuous functions $q_{i}(x)$ on $I$ and $q_{n}(x) \neq 0$ on $I$.

Let $a \in I$ and assume that $\phi(x, \lambda)$ is continuous in $\lambda$ and $\phi(a, \lambda) \neq 0$. Without loss of generality, we may take $\phi(a, \lambda)=1$. If $\phi(a, \lambda)=0$, we may take $\phi$ to satisfy $\left.\frac{\partial \phi(x, \lambda)}{\partial x}\right|_{x=a}=1$. Such $\phi$ always exists as one of the fundamental solutions of the initial-value problem [28].

We will focus on the case $J_{1}=[0, \infty)$ because the case $J_{1}=(-\infty, \infty)$ can be treated similarly. We assume that $\hat{f}$ has compact support of the form $[d, b], 0 \leq d<$ $b<\infty$. By translation, we may, without loss of generality, assume that the support of $\hat{f}$ is $[0, b]$.

Let $w(\lambda)>0$ be a weight function on $0 \leq \lambda \leq b$. Let $\left\{p_{n}(\lambda)\right\}_{n=0}^{\infty}$ be a complete orthonormal system of polynomials in $L^{2}([0, b), w(\lambda))$ with respect to the weight function $w(\lambda)$.

Consider $f(x)$, the integral transform (3.7) of $\hat{f}$ :

$$
f(x)=\int_{0}^{b} \hat{f}(\lambda) \phi(x, \lambda) d \lambda, \quad x \in I .
$$

Applying the operator $L$ to both sides of (3.9), we get

$$
p_{n}(L) f(x)=\int_{0}^{b} p_{n}(\lambda) \hat{f}(\lambda) \phi(x, \lambda) d \lambda .
$$


Moreover, we have $\left[p_{n}(L) f\right](a)=\int_{0}^{b} p_{n}(\lambda) \hat{f}(\lambda) d \lambda=\left\langle\hat{f}, p_{n}\right\rangle$. Thus, formally we have

$$
f(x)=\sum_{n=0}^{\infty} K^{n}[f](a) \psi_{n}(x),
$$

where the series converges pointwise for $x \in I$,

$$
\psi_{n}(x)=K^{n}[\psi](x)=\int_{0}^{b} p_{n}(\lambda) w(\lambda) \phi(x, \lambda) d \lambda, \quad x \in I,
$$

with

$$
\psi(x)=\int_{0}^{b} w(\lambda) \phi(x, \lambda) d \lambda,
$$

and $K^{n}[f](a)=\left[p_{n}(L) f\right](a)$ is the $n$-th chromatic derivative of $f$ associated with the integral transform (3.7) and the system of orthonormal polynomials $\left\{p_{n}\right\}$.

\section{Chromatic expansions in Weighted Bergman spaces}

In this section we derive chromatic series expansions for functions in weighted Bergman spaces. The expansions use chromatic derivatives associated with the Laguerre polynomials.

Let $\gamma>0, \mathbb{D}$ denote the open unit disk, and $\mathfrak{B}_{\gamma}$ denote the space of all analytic functions $f$ in $\mathbb{D}$ such that

$$
\|f\|_{\mathfrak{B}_{\gamma}}^{2}=\frac{\gamma}{\pi} \int_{\mathbb{D}}|f(z)|^{2}(1-z \bar{z})^{\gamma-1} d z<\infty .
$$

The space $\mathfrak{B}_{\gamma}$ is a Hilbert space with inner product

$$
\langle f, g\rangle=\frac{\gamma}{\pi} \int_{\mathbb{D}} f(z) \bar{g}(z)(1-z \bar{z})^{\gamma-1} d z .
$$

For $\gamma=1$, the space $\mathfrak{B}_{1}$ is the standard Bergman space, which will be denoted by $\mathfrak{B}$; see 8 . Let $f$ and $g$ have Taylor series

$$
f(z)=\sum_{n=0}^{\infty} a_{n} z^{n}, \quad g(z)=\sum_{n=0}^{\infty} b_{n} z^{n} .
$$

Then

$$
\begin{aligned}
\langle f, g\rangle_{\mathfrak{B}_{\gamma}} & =\frac{\gamma}{\pi} \sum_{m, n=0}^{\infty} a_{n} \bar{b}_{m} \int_{\mathbb{D}} z^{n} \bar{z}^{m}(1-z \bar{z})^{\gamma-1} d z \\
& =\frac{\gamma}{\pi} \sum_{m, n=0}^{\infty} a_{n} \bar{b}_{m} \int_{0}^{1} r^{m+n+1}\left(1-r^{2}\right)^{\gamma-1} d r \int_{0}^{2 \pi} e^{i(n-m) \theta} d \theta \\
& =2 \gamma \sum_{n=0}^{\infty} a_{n} \bar{b}_{n} \int_{0}^{1} r^{2 n+1}\left(1-r^{2}\right)^{\gamma-1} d r \\
& =\gamma \sum_{n=0}^{\infty} a_{n} \bar{b}_{n} \int_{0}^{1} x^{n}(1-x)^{\gamma-1} d x \\
& =\gamma \sum_{n=0}^{\infty} a_{n} \bar{b}_{n} B(n+1, \gamma)
\end{aligned}
$$


where $B(a, b)$ is the beta function given by

$$
B(a, b)=\frac{\Gamma(a) \Gamma(b)}{\Gamma(a+b)} .
$$

If we use the notation

$$
\left(\begin{array}{l}
a \\
b
\end{array}\right)=\frac{\Gamma(a+1)}{\Gamma(b+1) \Gamma(a-b+1)}
$$

we obtain

$$
\langle f, g\rangle_{\mathfrak{B}_{\gamma}}=\sum_{n=0}^{\infty} \frac{a_{n} \bar{b}_{n}}{\left(\begin{array}{l}
n+\gamma \\
n
\end{array}\right)}
$$

Hence

$$
\|f\|_{\mathfrak{B}_{\gamma}}^{2}=\sum_{n=0}^{\infty} \frac{\left|a_{n}\right|^{2}}{\left(\begin{array}{l}
n+\gamma \\
n
\end{array}\right)}
$$

Let

$$
u_{n}(z)=\sqrt{\left(\begin{array}{l}
n+\gamma \\
n
\end{array}\right)} z^{n} .
$$

From (4.2), it immediately follows that $\left\{u_{n}(z)\right\}_{n=0}^{\infty}$ is an orthonormal basis of $\mathfrak{B}_{\gamma}$.

Let $\tilde{L}_{n}^{\gamma}$ denote the Laguerre polynomial of degree $n$ defined by

$$
\tilde{L}_{n}^{\gamma}(x)=\frac{x^{-\alpha} e^{x}}{n !}\left(\frac{d}{d x}\right)^{n}\left(x^{n+\alpha} e^{-x}\right), \quad n=0,1, \cdots .
$$

and define

$$
\psi_{n}(q)=\frac{1}{\left(\begin{array}{l}
n+\gamma \\
n
\end{array}\right)} e^{-q / 2} \tilde{L}_{n}^{\gamma}(q)=e^{-q / 2} L_{n}^{\gamma}(q),
$$

where $L_{n}^{\gamma}(q)$ are the normalized Laguerre polynomials

$$
L_{n}^{\gamma}(q)=\tilde{L}_{n}^{\gamma}(q) /\left(\begin{array}{l}
n+\gamma \\
n
\end{array}\right)
$$

such that

$$
\int_{0}^{\infty} L_{m}^{\gamma}(q) L_{n}^{\gamma}(q) e^{-q} \frac{q^{\gamma}}{\Gamma(\gamma+1)} d q=\delta_{m, n}
$$

Therefore, if we set

$$
d \mu=\frac{q^{\gamma}}{\Gamma(\gamma+1)} d q
$$

it follows that

$$
\left\langle\psi_{m}, \psi_{n}\right\rangle_{d \mu}=\int_{\mathbb{R}^{+}} \psi_{m}(q) \psi_{n}(q) d \mu=\delta_{m, n} .
$$

The set $\left\{\psi_{n}(q)\right\}_{n=0}^{\infty}$ is an orthonormal basis of $L^{2}\left(\mathbb{R}^{+}, d \mu\right)$, and hence, for any $F \in L^{2}\left(\mathbb{R}^{+}, d \mu\right)$, we have

$$
F(q)=\sum_{n=0}^{\infty} \hat{F}_{n} \psi_{n}(q), \quad \text { with } \hat{F}_{n}=\left\langle F, \psi_{n}\right\rangle_{d \mu},
$$


and

$$
\|F\|_{d \mu}^{2}=\sum_{n=0}^{\infty}\left|\hat{F}_{n}\right|^{2}<\infty .
$$

Moreover, for $F, G \in L^{2}\left(\mathbb{R}^{+}, d \mu\right)$ we have

$$
\langle F, G\rangle_{d \mu}=\sum_{n=0}^{\infty} F_{n} \bar{G}_{n}<\infty .
$$

Definition 4.1. Let $f \in C^{\infty}\left(\mathbb{R}^{+}\right)$and

$$
\mathcal{L}=-(1-z)^{1-\gamma} \frac{d}{d z}(1-z)^{1+\gamma} .
$$

We define the $n$-chromatic derivative of $f$ with respect to the Laguerre polynomials as $K_{\gamma}^{n} f(z)=L_{n}^{\gamma}(\mathcal{L}) f(z)$, where $L_{n}^{\gamma}(\mathcal{L})$ has the same meaning as in Section 3

We have the following theorem.

Theorem 4.2. For any $f \in \mathfrak{B}_{\gamma}$, we have the expansion

$$
f(z)=\sum_{n=0}^{\infty} K_{\gamma}^{n} f(0) K_{\gamma}^{n} \psi(z),
$$

where $K_{\gamma}^{n}$ is the $n$-th chromatic derivative with respect to the Laguerre polynomials and

$$
\psi(z)=\int_{\mathbb{R}^{+}} \frac{1}{(1-z)^{\gamma+1}} \exp \left\{-\frac{q}{(1-z)}\right\} d \mu .
$$

The series converges uniformly on compact subsets of the open unit disk.

Proof. Let

$$
\begin{aligned}
k(z, q) & =\sum_{n=0}^{\infty} u_{n}(z) \psi_{n}(q)=e^{-q / 2} \sum_{n=0}^{\infty} z^{n} \tilde{L}_{n}^{\gamma}(q) \\
& =e^{-q / 2} e^{-q z /(1-z)}(1-z)^{-\gamma-1} \\
& =(1-z)^{-\gamma-1} \exp \left\{-\frac{q(1+z)}{2(1-z)}\right\}, \quad|z|<1
\end{aligned}
$$

where the series converges to $k(z, q)$ in $L^{2}\left(\mathbb{R}^{+}, d \mu\right)$ as a function of $q$ for every $z \in \mathbb{D}$ and uniformly on compact subsets of the open unit disk as a function of $z$; see [24, p. 77]. Let $F \in L^{2}\left(\mathbb{R}^{+}, d \mu\right)$ and consider the integral transform

$$
\begin{aligned}
\mathcal{T}[F](z)=f(z) & =\int_{\mathbb{R}^{+}} F(q) k(z, q) d \mu(q) \\
& =\int_{\mathbb{R}^{+}} \frac{F(q)}{(1-z)^{\gamma+1}} \exp \left\{-\frac{q(1+z)}{2(1-z)}\right\} \frac{q^{\gamma}}{\Gamma(\gamma+1)} d q .
\end{aligned}
$$

It is easy to see that if $F \in L^{2}\left(\mathbb{R}^{+}, d \mu\right)$, then $f(z)=\mathcal{T}[F](z) \in \mathfrak{B}_{\gamma}$. For, if $F=\sum_{n=0}^{\infty} \hat{F}_{n} \psi_{n}(q)$, then

$$
f(z)=\sum_{n=0}^{\infty} \hat{F}_{n} u_{n}(z)=\sum_{n=0}^{\infty} \hat{F}_{n} \sqrt{\left(\begin{array}{l}
n+\gamma \\
n
\end{array}\right)} z^{n},
$$


where interchanging the summation and integration is permissable for every fixed $z$. Moreover, the series converges uniformly on any compact subset of $\mathbb{D}$ because for all $|z| \leq \delta<1$ we have

$$
\left|\sum_{n=N}^{\infty} \hat{F}_{n} u_{n}(z)\right|^{2} \leq\left(\sum_{n=N}^{\infty}\left|\hat{F}_{n}\right|^{2}\right)\left(\sum_{n=N}^{\infty}\left(\begin{array}{l}
n+\gamma \\
n
\end{array}\right) \delta^{2 n}\right) \rightarrow 0
$$

as $N \rightarrow \infty$. By (4.3), we have

$$
\|f\|_{\mathfrak{B}_{\gamma}}^{2}=\sum_{n=0}^{\infty} \frac{\left|\hat{F}_{n}\right|^{2}}{\left(\begin{array}{l}
n+\gamma \\
n
\end{array}\right)}\left(\begin{array}{l}
n+\gamma \\
n
\end{array}\right)=\sum_{n=0}^{\infty}\left|\hat{F}_{n}\right|^{2}<\infty,
$$

which implies that $f \in \mathfrak{B}_{\gamma}$.

Now in view of Section 3 and (4.5), it is easy to see that

$$
\mathcal{L} f(z)=\int_{\mathbb{R}^{+}} q F(q) k(z, q) d \mu
$$

hence

$$
L_{n}^{\gamma}(\mathcal{L}) f(z)=\int_{\mathbb{R}^{+}} L_{n}^{\gamma}(q) F(q) k(z, q) d \mu=K_{\gamma}^{n} f(z) .
$$

Since $k(0, q)=e^{-q / 2}$, it follows that

$$
\begin{aligned}
L_{n}^{\gamma}(\mathcal{L}) f(0) & =\int_{\mathbb{R}^{+}} L_{n}^{\gamma}(q) e^{-q / 2} F(q) d \mu \\
& =\left\langle F, \psi_{n}\right\rangle_{d \mu}=K_{\gamma}^{n} f(0) .
\end{aligned}
$$

Thus,

$$
f(z)=\sum_{n=0}^{\infty} K_{\gamma}^{n} f(0) \int_{\mathbb{R}^{+}} \psi_{n}(q) k(z, q) d \mu .
$$

But since

$$
\psi(z)=\int_{\mathbb{R}^{+}} \frac{1}{(1-z)^{\gamma+1}} \exp \left\{-\frac{q}{(1-z)}\right\} d \mu=\int_{\mathbb{R}^{+}} e^{-q / 2} k(z, q) d \mu,
$$

one can show that

$$
\begin{aligned}
L_{n}^{\gamma}(\mathcal{L}) \psi(z) & =\int_{\mathbb{R}^{+}} L_{n}^{\gamma}(q) e^{-q / 2} k(z, q) d \mu \\
& =\int_{\mathbb{R}^{+}} \psi_{n}(q) k(z, q) d \mu=K_{\gamma}^{n} \psi(z) .
\end{aligned}
$$

By substituting (4.10) into (4.9), we obtain (4.4).

\section{ORTHOGONAL POLYNOMIAL EXPANSIONS IN SEVERAL VARIABLES}

5.1. Orthogonal polynomials in several variables. Let $\mathbb{N}$ denote the set of nonnegative integers and $\alpha$ be a multi-index $\alpha=\left(\alpha_{1}, \ldots, \alpha_{d}\right) \in \mathbb{N}^{d}$. We use the notation

$$
\alpha !=\alpha_{1} ! \alpha_{2} ! \ldots \alpha_{d} !, \quad|\alpha|=\alpha_{1}+\ldots+\alpha_{d}
$$


and $\delta_{\alpha, \beta}=\delta_{\alpha_{1}, \beta_{1}} \ldots \delta_{\alpha_{d}, \beta_{d}}$. For $x=\left(x_{1}, \ldots, x_{d}\right) \in \mathbb{R}^{d}$, we define the monomial $x^{\alpha}=x_{1}^{\alpha_{1}} \ldots x_{d}^{\alpha_{d}}$ and $|\alpha|$ is the degree of $x^{\alpha}$. A polynomial $P$ in $d$ variables is a linear combination of the form

$$
P(x)=\sum_{\alpha} c_{\alpha} x^{\alpha}
$$

where $c_{\alpha}$ are complex numbers. We denote the set of all polynomials in $d$ variables by $\Pi^{d}$ and the set of all polynomials of degree at most $n$ by $\Pi_{n}^{d}$. The set of all homogenous polynomials of degree $n$ will be denoted by $P_{n}^{d}$ :

$$
P_{n}^{d}=\left\{P: P(x)=\sum_{|\alpha|=n} c_{\alpha} x^{\alpha}\right\} .
$$

Every polynomial in $d$ variables can be written as a linear combination of homogenous polynomials

$$
P(x)=\sum_{k=0}^{n} \sum_{|\alpha|=k} c_{\alpha} x^{\alpha} .
$$

It is known that the dimension $r_{n}^{d}$ of $P_{n}^{d}$ is

$$
r_{n}^{d}=\left(\begin{array}{l}
n+d-1 \\
n
\end{array}\right) .
$$

For a fixed $d$, we may write $r_{n}=r_{n}^{d}$. We shall use the lexicographic order, i.e., $\alpha>\beta$, if the first nonzero entry in the difference $\alpha-\beta=\left(\alpha_{1}-\beta_{1}, \alpha_{2}-\beta_{2}, \ldots, \alpha_{d}-\beta_{d}\right)$ is positive.

If $\langle$,$\rangle is an inner product on \Pi^{d}$, we say that a polynomial $P$ is orthogonal to a polynomial $Q$ if $\langle P, Q\rangle=0$. A polynomial $P$ is called an orthogonal polynomial if $P$ is orthogonal to all polynomials of lower degree, i.e., $\langle P, Q\rangle=0$ for all $Q \in \Pi^{d}$ with $\operatorname{deg} Q<\operatorname{deg} P$. Denote by $V_{n}^{d}$ the space of orthogonal polynomials of degree exactly $n$ :

$$
V_{n}^{d}=\left\{P \in \Pi_{n}^{d}:\langle P, Q\rangle=0, \text { for all } Q \in \Pi_{n-1}^{d}\right\} ;
$$

the dimension of $V_{n}^{d}$ is the same as that of $P_{n}^{d}$.

A multi-sequence $s: \mathbb{N}^{d} \rightarrow \mathbb{R}$ is written as $s=\left(s_{\alpha}\right)_{\alpha \in \mathbb{N}^{d}}$, and for each multisequence we define a linear functional on $\Pi^{d}$ by

$$
\mathcal{L}\left(x^{\alpha}\right)=s_{\alpha}, \quad \alpha \in \mathbb{N}^{d} .
$$

Let the elements of the set $\left\{\alpha \in \mathbb{N}^{d}:|\alpha|=n\right\}$ be arranged as $\alpha^{(1)}, \alpha^{(2)}, \ldots, \alpha^{\left(r_{n}\right)}$ according to th lexicographic order. Let $\mathbf{x}^{\mathbf{n}}$ denote the column vector

$$
\mathbf{x}^{n}=\left(x^{\alpha}\right)_{|\alpha|=n}=\left(x^{\alpha(j)}\right)_{j=1}^{r_{n}} ;
$$

that is, $\mathbf{x}^{n}$ is a vector whose elements are the monomials $x^{\alpha}$ for $|\alpha|=n$, arranged in lexicographic order.

Define the vector moments $\mathbf{s}_{k}=\mathcal{L}\left(\mathbf{x}^{k}\right)$ and

$$
\mathbf{s}_{(k)+(j)}=\mathcal{L}\left(\mathbf{x}^{k}\left(\mathbf{x}^{j}\right)^{T}\right),
$$

which is a matrix of size $r_{k}^{d} \times r_{j}^{d}$ whose elements are $\mathcal{L}\left(x^{\alpha+\beta}\right)$ for $|\alpha|=k,|\beta|=j$.

Define the moment matrix

$$
M_{n, d}=\left(s_{(k)+(j)}\right)_{k, j=0}^{n} \text { and } \Delta_{n, d}=\operatorname{det} M_{n, d}
$$


whose elements are $\mathcal{L}\left(x^{\alpha+\beta}\right)$ for $|\alpha| \leq n,|\beta| \leq n$. If $\left\{P_{\alpha}\right\}_{|\alpha|=n}$ is a sequence of polynomials in $\Pi_{n}^{d}$, we get the column polynomial vector $\mathbb{P}_{n}=\left(P_{\alpha(1)}, \ldots, P_{\alpha^{\left(r_{n}\right)}}\right)^{T}$, where $\alpha^{(1)}, \ldots, \alpha^{\left(r_{n}\right)}$ is the lexicographic order in $\left\{\alpha \in \mathbb{N}^{d}:|\alpha|=n\right\}$. For more details on the subsequent discussion, see [7].

Definition 5.1. Let $\mathcal{L}$ be a moment functional. A sequence of polynomials $\left\{P_{\alpha}\right\}_{|\alpha|=n}$ in $\Pi_{n}^{d}$ is said to be orthogonal with respect to $\mathcal{L}$ if

$$
\mathcal{L}\left(\mathbf{x}^{m} \mathbb{P}_{n}^{T}\right)=0 \text { for } n>|m|, \text { and } \mathcal{L}\left(\mathbf{x}^{n} \mathbb{P}_{n}^{T}\right)=s_{n},
$$

where $s_{n}$ is an invertible matrix of size $r_{n}^{d} \times r_{n}^{d}$.

It is known that if $\mathcal{L}$ is a moment functional and $\mathbb{P}_{n}$ is orthogonal as defined before, then $\left\{\mathbb{P}_{0}, \mathbb{P}_{1}, \cdots, \mathbb{P}_{n}\right\}$ is a basis for $\Pi_{n}^{d}$. Hence, there exist matrices $\mathbf{c}_{k}$ of size $r_{n}^{d} \times r_{k}^{d}$ such that

$$
\mathbf{x}^{n}=\mathbf{c}_{n} \mathbb{P}_{n}+\mathbf{c}_{n-1} \mathbb{P}_{n-1}+\ldots+\mathbf{c}_{0} \mathbb{P}_{0} .
$$

Moreover, for a given moment functional $\mathcal{L}$, a system of orthogonal polynomials exists if and only if $\Delta_{n, d} \neq 0$.

It is known [7] that if $\mathcal{L}$ is positive definite, then $\Delta_{n, d}>0$ and there exists a system of orthogonal polynomials with respect to $\mathcal{L}$. In fact, in this case there exists an orthonormal basis with respect to $\mathcal{L}$; that is, there exists a sequence of vector polynomials $\left\{\mathbb{P}_{n}\right\}$ such that

$$
\mathcal{L}\left(\mathbb{P}_{m} \mathbb{P}_{n}^{T}\right)=0 \text { if } m \neq n, \quad \mathcal{L}\left(\mathbb{P}_{n} \mathbb{P}_{n}^{T}\right)=I_{r_{n}},
$$

where $I_{r_{n}}$ is the identity matrix of size $r_{n}^{d} \times r_{n}^{d}$.

Let $M$ denote the set of nonnegative Borel measures on $\mathbb{R}^{d}$ having moments of all orders. Thus, $\mu \in M$ if

$$
\int_{\mathbb{R}^{d}}|x|^{\alpha} d \mu<\infty \text { for all } \alpha \in \mathbb{N}^{d}
$$

and we have the moments of $\mu$,

$$
s_{\alpha}=\int_{\mathbb{R}^{d}} x^{\alpha} d \mu
$$

For such a measure $\mu \in M$, we have a moment functional $\mathcal{L}$ defined for polynomials $P \in \Pi^{d}$ by

$$
\mathcal{L}(P)=\int_{\mathbb{R}^{d}} P(x) d \mu(x) .
$$

If $d \mu(x)=W(x) d x$ and $W(x)$ is a nonnegative weight function, then $\mathcal{L}$ is positive definite, i.e., $\mathcal{L}\left(P^{2}\right)>0$ for any $0 \neq P \in \Pi^{d}$. It is known that if $s=\left(s_{\alpha}\right)$ is a multi-sequence, then there exists $\mu \in M$ such that $s_{\alpha}=\int x^{\alpha} d \mu(x)$ if and only if the associated moment functional $\mathcal{L}$ is nonnegative on the set of nonnegative polynomials.

5.2. Orthogonal polynomial expansions. Let $\mathcal{L}$ be a positive moment functional (hence positive definite) and let $\left\{\mathbb{P}_{n}\right\}$ be the system of orthonormal polynomials associated with it. There exists a measure $\mu \in M$ such that

$$
\mathcal{L}\left(x^{\alpha}\right)=s_{\alpha}=\int_{\mathbb{R}^{d}} x^{\alpha} d \mu(x) .
$$


We assume that $\mu$ is absolutely continuous so that $d \mu=W(x) d x$, with $W$ being nonnegative so that $\mathcal{L}_{W}(f)=\int f(x) W(x) d x$ is positive definite. We also assume that for some $c>0$,

$$
\int_{\mathbb{R}^{d}} e^{c\|x\|} W(x) d(x)<\infty,
$$

so that polynomials are dense in $L^{2}(d \mu)$, [7]. Condition (5.2) is satisfied, for example, if $\mu$ is compactly supported.

We adopt the following notation. If $P\left(x_{1}, \ldots, x_{d}\right)$ is a polynomial in $x_{1}, \ldots, x_{d}$, then the polynomial $P\left(\frac{\partial}{\partial x_{1}}, \ldots, \frac{\partial}{\partial x_{d}}\right)$ will be denoted by $P\left(\frac{\partial}{\partial x}\right)$, where $x^{\alpha}$ is replaced by $\frac{\partial^{|\alpha|}}{\partial x^{\alpha}}$ where $\alpha$ is a multi-index. More explicitly if $\alpha=\left(\alpha_{1}, \ldots, \alpha_{d}\right)$, then

$$
x^{\alpha}=x_{1}^{\alpha_{1}} x_{2}^{\alpha_{2}} \cdots x_{d}^{\alpha_{d}}, \quad|\alpha|=\alpha_{1}+\alpha_{2}+\cdots+\alpha_{d}, \alpha_{i} \in \mathbb{N},
$$

is replaced by

$$
\frac{\partial^{|\alpha|}}{\partial x_{1}^{\alpha_{1}} \partial x_{2}^{\alpha_{2}} \cdots \partial x_{d}^{\alpha_{d}}} .
$$

Let $\left\{P_{\alpha}^{n}\right\}_{|\alpha|=0}^{\infty}$ denote the sequence of orthonormal polynomials with respect to $\mathcal{L}_{W}$. Let $L_{W}^{2}\left(\mathbb{R}^{d}\right)$ denote the space of all square integrable functions with respect to the weight function $W$. For any function $f \in L_{W}^{2}\left(\mathbb{R}^{d}\right)$, consider its generalized Fourier expansion with respect to $\left\{P_{\alpha}^{n}\right\}_{|\alpha|=0}^{\infty}$,

$$
f \backsim \sum_{n=0}^{\infty} \sum_{|\alpha|=n} c_{\alpha}^{n}(f) P_{\alpha}^{n}, \quad \text { with } \quad c_{\alpha}^{n}(f)=\left\langle f, P_{\alpha}^{n}\right\rangle_{W}=\int f(x) P_{\alpha}^{n}(x) W(x) d x .
$$

If we use the vector notation, we have

$$
f \backsim \sum_{n=0}^{\infty} \mathbf{c}_{n}^{T}(f) \mathbb{P}_{n}, \quad \text { with } \quad \mathbf{c}_{n}(f)=\left\langle f, \mathbb{P}_{n}\right\rangle_{W}=\int f(x) \mathbb{P}_{n}(x) W(x) d x ;
$$

here $\mathbf{c}_{n}$ is a column vector with components $c_{\alpha}^{n}$ and $|\alpha|=n$.

\section{Multi-Dimensional Chromatic Derivatives}

Let $z=\left(z_{1}, z_{2}, \cdots, z_{d}\right) \in \mathbb{C}^{d}$ and define the inner product

$$
\langle z, w\rangle=\sum_{k=1}^{d} z_{k} \bar{w}_{k}, z, w \in \mathbb{C}^{d} \text { so that }\|z\|^{2}=\sum_{k=1}^{d}\left|z_{k}\right|^{2} .
$$

If $z_{k}=x_{k}+i y_{k}$, define $\Re z=\left(x_{1}, x_{2}, \cdots, x_{d}\right)$ and $\Im z=\left(y_{1}, y_{2}, \cdots, y_{d}\right)$ so that $z=\Re z+i \Im z$. Moreover,

$$
\|\Re z\|^{2}=\sum_{k=1}^{d} x_{k}^{2}, \quad\|\Im z\|^{2}=\sum_{k=1}^{d} y_{k}^{2} .
$$

For every real $a>0$, we let $S_{d}(a)=\left\{z \in \mathbb{C}^{d}:\|\Im(z)\|<a\right\}$.

Definition 6.1. Let $f: \mathbb{C}^{d} \rightarrow \mathbb{C}$; the $n$-th chromatic derivatives $\mathbb{K}^{n}(f)$ of $f(z)$ with respect to the polynomials $\left\{P_{\alpha}^{n}\right\}$ is defined as

$$
\mathbb{K}^{n}(f)=\mathbb{P}_{n}\left(-i \frac{\partial}{\partial z}\right)(f)
$$

where $\mathbb{P}_{n}$ is the column vector defined before. 
Hence, the $n$-th chromatic derivative of $f$ is a column vector with $r_{n}^{d}$ components, with each component being a linear combination of partial derivatives. It is easy to check that if $\omega \in \mathbb{R}^{d}$ is fixed, then 1

$$
K_{\alpha}^{n}\left(e^{i\langle\omega, z\rangle}\right)=P_{\alpha}^{n}(\omega) e^{i\langle\omega, z\rangle} .
$$

Proposition 6.2. Let $\varphi(\omega) \in L_{W}^{2}\left(\mathbb{R}^{d}\right)$ and define a corresponding function $f_{\varphi}$ : $S_{d}(c / 2) \rightarrow \mathbb{C}$ by a $W(\omega)$-weighted Fourier transform of $\varphi$ :

$$
f_{\varphi}(z)=\int_{\mathbb{R}^{d}} \varphi(\omega) e^{i\langle\omega, z\rangle} W(\omega) d \omega .
$$

Then $f_{\varphi}(z)$ is analytic on $S_{d}(c / 2)$, and for all $n$ and $z \in S_{d}(c / 2)$,

$$
\mathbb{K}^{n}\left[f_{\varphi}\right](z)=\int_{\mathbb{R}^{d}} \mathbb{P}_{n}(\omega) \varphi(\omega) e^{i\langle\omega, z\rangle} W(\omega) d \omega .
$$

For the proof, see [12]. It is known that if condition (5.2) holds, then $\left\{\mathbb{P}_{n}(\omega)\right\}$ is a complete system in $L_{W}^{2}\left(\mathbb{R}^{d}\right)$.

Proposition 6.3. Let $\varphi(\omega) \in L_{W}^{2}\left(\mathbb{R}^{d}\right)$; if for some fixed $u \in S_{d}(c / 2)$ the function $\varphi(\omega) e^{i\langle\omega, u\rangle}$ belongs to $L_{W}^{2}\left(\mathbb{R}^{d}\right)$, then in $L_{W}^{2}\left(\mathbb{R}^{d}\right)$ we have

$$
\varphi(\omega) e^{i\langle\omega, u\rangle}=\sum_{n=0}^{\infty}\left[\mathbb{K}^{n}\left[f_{\varphi}\right](u)\right]^{T} \mathbb{P}_{n}(\omega),
$$

and for $f_{\varphi}$ given by (6.2) we have

$$
\sum_{n=0}^{\infty} \sum_{|\alpha|=n}\left|K_{\alpha}^{n}\left[f_{\varphi}\right](u)\right|^{2}=\left\|\varphi(\omega) e^{i\langle\omega, u\rangle}\right\|_{W}^{2}<\infty .
$$

Proof. Let $f_{\varphi}$ be given by (6.2). By Proposition 6.2, (6.3) holds. However, if $\varphi(\omega) e^{i\langle\omega, u\rangle}$ belongs to the space $L_{W}^{2}\left(\mathbb{R}^{n}\right)$, then (6.3) asserts that the projection of $\varphi(\omega) e^{i\langle\omega, u\rangle}$ onto the vector $\mathbb{P}_{n}(\omega)$ is equal to $\mathbb{K}^{n}\left[f_{\varphi}\right](u)$ :

$$
\left\langle\varphi(\omega) e^{i\langle\omega, u\rangle}, \mathbb{P}_{n}(\omega)\right\rangle_{W}=\mathbb{K}^{n}\left[f_{\varphi}\right](u) .
$$

Since $\left\{\mathbb{P}_{n}(\omega)\right\}_{n \in \mathbb{N}}$ is a complete orthonormal system in $L_{W}^{2}\left(\mathbb{R}^{d}\right)$, (6.6) implies (6.4), and Parseval's Theorem implies (6.5).

As a corollary, we have

Corollary 6.4. For every $\varphi(\omega) \in L_{W}^{2}\left(\mathbb{R}^{d}\right)$ and every $u \in \mathbb{R}^{d}$, equality (6.4) holds and

$$
\sum_{n=0}^{\infty} \sum_{|\alpha|=n}\left|K_{\alpha}^{n}\left[f_{\varphi}\right](u)\right|^{2}=\|\varphi(\omega)\|_{W}^{2} .
$$

Thus, the sum $\sum_{n=0}^{\infty} \sum_{|\alpha|=n}\left|K_{\alpha}^{n}\left[f_{\varphi}\right](u)\right|^{2}$ is independent of $u \in \mathbb{R}^{d}$.

Proof. If $u \in \mathbb{R}^{d}$, then $\varphi(\omega) e^{i\langle\omega, u\rangle} \in L_{W}^{2}\left(\mathbb{R}^{d}\right)$ and $\left\|\varphi(\omega) e^{i\langle\omega, u\rangle}\right\|_{W}^{2}=\|\varphi(\omega)\|_{W}^{2} ;$ thus, Proposition 6.3 applies.

\footnotetext{
${ }^{1}$ If $x, \omega \in \mathbb{R}^{d}$ are both real vectors, then we denote their scalar product by $x . \omega$. If at least one of $u, z$ is complex we denote their scalar product by $\langle u, z\rangle$.
} 
Definition 6.5. Let $z \in S_{d}(c / 2)$ and define

$$
\psi(z)=\int_{\mathbb{R}^{d}} e^{i\langle\omega, z\rangle} W(\omega) d \omega,
$$

and more generally

$$
K_{\alpha}^{n}(\psi(z))=\psi_{\alpha}^{n}(z)=\int_{\mathbb{R}^{d}} P_{\alpha}^{n}(\omega) e^{i\langle\omega, z\rangle} W(\omega) d \omega ;
$$

we may also use the vector notation $\boldsymbol{\Psi}_{n}(z)=\left(\psi_{\alpha}^{n}(z)\right)_{|\alpha|=n}$.

Note that Proposition 6.2 implies that the integrals in the definitions of $\psi(z)$ and $\psi_{\alpha}^{n}(z)$ are finite.

Corollary 6.6. Let $\varepsilon>0$; then for all $z \in S\left(\frac{c}{2}-\varepsilon\right)$

$$
\sum_{n=0}^{\infty} \sum_{|\alpha|=n}\left|\psi_{\alpha}^{n}(z)\right|^{2}<\left\|e^{\left(\frac{c}{2}-\varepsilon\right)\|\omega\|}\right\|_{W}^{2}<\infty .
$$

Proof. We apply Proposition 6.3 with $\varphi(\omega)=1$, in which case $f_{\varphi}(z)=\psi(z)$, and by using (6.5), we obtain

$$
\sum_{n=0}^{\infty} \sum_{|\alpha|=n}\left|\psi_{\alpha}^{n}(z)\right|^{2}=\left\|e^{i\langle\omega, z\rangle}\right\|_{W}^{2} \leq\left\|e^{\|\omega\|\|\Im(z)\|}\right\|_{W}^{2}<\left\|e^{\left(\frac{c}{2}-\varepsilon\right)\|\omega\|}\right\|_{W}^{2}<\infty .
$$

The last inequality follows from (5.2).

Definition 6.7. We denote by $\Lambda_{W}^{2}$ the vector space of functions $f: S_{d}(c / 2) \rightarrow \mathbb{C}$ which are analytic on $S_{d}(c / 2)$ and satisfy $\sum_{n=0}^{\infty} \sum_{|\alpha|=n}\left|K_{\alpha}^{n}\left[f_{\varphi}\right](0)\right|^{2}<\infty$. The chromatic series expansion of a function $f \in \Lambda_{W}^{2}$ is given by the following formal series:

$$
f(z) \sim \sum_{n=0}^{\infty}\left[\mathbb{K}^{n}(f)(u)\right]^{T} \Psi_{n}(z-u) .
$$

Proposition 6.8. The mapping

$$
f(z) \mapsto \varphi_{f}(\omega)=\sum_{n=0}^{\infty}\left[\mathbb{K}^{n}[f](0)\right]^{T} \mathbb{P}_{n}(\omega)
$$

is an isomorphism between the vector spaces $\Lambda_{W}^{2}$ and $L_{W}^{2}\left(\mathbb{R}^{d}\right)$, and its inverse is given by (6.2).

Definition 6.9. For $f \in \Lambda_{W}^{2}$, we denote the corresponding $\varphi_{f}(\omega)$ given by (6.12) by $\mathcal{F}_{W}[f](\omega)$; thus, for $z \in S_{d}(c / 2)$,

$$
f(z)=\int_{\mathbb{R}^{d}} \mathcal{F}_{W}[f](\omega) e^{i\langle\omega, z\rangle} W(\omega) d \omega .
$$

Proposition 6.8 and Corollary 6.4 imply the following main result.

Theorem 6.10. For all $f \in \Lambda_{W}^{2}$ and all $u \in \mathbb{R}^{d}$, the sum $\sum_{n=0}^{\infty} \sum_{|\alpha|=n}\left|K_{\alpha}^{n}\left[f_{\varphi}\right](u)\right|^{2}$ converges and is independent of $u$. Moreover,

$$
f(z)=\sum_{n=0}^{\infty}\left[\mathbb{K}^{n}(f)(u)\right]^{T} \Psi_{n}(z-u) .
$$


Remark. The most efficient system of orthogonal polynomials for one to use to generate chromatic derivatives will depend on the shape of the support of the Fourier transform $\mathcal{F}_{W}[f]$ of $f$. If the support of $\mathcal{F}_{W}[f]$ is the hypercube $D=[-1,1]^{d}$, we may use the $d$-dimensional Jacobi polynomials

$$
P_{\alpha}^{(a, b)}(x)=P_{\alpha_{1}}^{\left(a_{1}, b_{1}\right)}\left(x_{1}\right) \cdots P_{\alpha_{d}}^{\left(a_{d}, b_{d}\right)}\left(x_{d}\right), \quad|\alpha|=n,
$$

with weight function

$$
W^{(a, b)}(x)=\prod_{i=1}^{d}\left(1-x_{i}\right)^{a_{i}}\left(1+x_{i}\right)^{b_{i}},
$$

where $a=\left(a_{1}, a_{2}, \cdots, a_{d}\right), b=\left(b_{1}, b_{2}, \cdots, b_{d}\right)$ and $P_{n}^{(\alpha, \beta)}(x)$ is the normalized Jacobi polynomial of degree $n$ with parameters $\alpha, \beta$. See [31 for the definition of the Jacobi, Laguerre, and Hermite polynomials.

\section{The Bargmann-Segal-Foch SPACE}

In 1928, V. Fock 10 introduced an operator solution of a commutation rule that appeared in quantum mechanics. In 1961, V. Bargmann [1 3] investigated in greater detail the function space $\mathfrak{F}$ on which Foch's solution is realized. He also studied the relationship between the space $\mathfrak{F}$, which is called the Bargmann-SegalFoch space, and the Hilbert space of square integrable functions $L^{2}\left(\mathbb{R}^{n}\right)$. That relationship was established using an integral transform that is now known as the Bargmann transform. The Bargmann transform resurfaced again in recent years because of its connection with other important transforms such as the Gabor and Zak transforms [20,21]. In this section we introduce the Bargmann-Segal-Foch space and some of its properties that will be used later.

Definition 7.1. Let $d \mu_{n}(z)=\rho_{n} d^{n} x d^{n} y, \rho_{n}=(\pi)^{-n} \exp \left(-\|z\|^{2}\right)$. The BargmannSegal-Foch space, $\mathfrak{F}$, consists of all entire functions $F(z)$ in $\mathbb{C}^{n}$ such that

$$
\|F\|_{\mathfrak{F}}^{2}=\int_{\mathbb{C}^{n}}|F(z)|^{2} d \mu_{n}(z)<\infty .
$$

It is a Hilbert space with inner product defined by

$$
\langle F, G\rangle_{\mathfrak{F}}=\int_{\mathbb{C}^{n}} F(z) \bar{G}(z) d \mu_{n}(z)<\infty,
$$

and hence with norm

$$
\|F\|_{\mathfrak{F}}^{2}=\int_{\mathbb{C}^{n}}|F(z)|^{2} d \mu(z) .
$$

The inner product can also be defined in terms of the Taylor series coefficients of $F$ and $G$. If $k=\left(k_{1}, \cdots, k_{n}\right), m=\left(m_{1}, \cdots, m_{n}\right)$ are multi-indices with $k_{i}, m_{i} \in \mathbb{N}$ and

$$
F(z)=\sum_{|k|=0} a_{k} z^{k} \text { and } G(z)=\sum_{|m|=0}^{\infty} b_{m} z^{m},
$$

$z^{k}=z_{1}^{k_{1}} z_{2}^{k_{2}} \cdots z_{n}^{k_{n}}$ and $z_{k}=r_{k} e^{i \theta_{k}}, k=1,2, \cdots, n$, then

$$
\langle F, G\rangle_{\mathfrak{F}}=\pi^{-n} \sum_{k, m} a_{k} \bar{b}_{m} B_{k, m}
$$


where

$$
B_{k, m}=\prod_{j=1}^{n}\left\{\left(\int_{0}^{2 \pi} e^{i\left(k_{j}-m_{j}\right) \theta_{j}} d \theta_{j}\right)\left(\int_{0}^{\infty} r_{j}^{k_{j}+m_{j}+1} e^{-r_{j}^{2}} d r_{j}\right)\right\} .
$$

Also, since

it is easily seen that

$$
\int_{0}^{\infty} r^{2 m+1} e^{-r^{2}} d r=\frac{m !}{2}
$$

$$
B_{k, m}= \begin{cases}0 & \text { if } k \neq m \\ \pi^{n} m ! & \text { if } k=m\end{cases}
$$

Hence

$$
\langle F, G\rangle_{\mathfrak{F}}=\sum_{|m|=0}^{\infty} a_{m} \bar{b}_{m} m !
$$

and

$$
\|F\|_{\mathfrak{F}}^{2}=\sum_{|m|=0}^{\infty}\left|a_{m}\right|^{2} m !
$$

Interchanging the integration and the summation signs is possible because of the uniform convergence.

Lemma 7.2. The set $\left\{u_{m}(z)=z^{m} / \sqrt{m !}\right\}_{|m|=0}^{\infty}$ is an orthonormal basis of $\mathfrak{F}$.

Proof. From (17.2) it follows that the set $\left\{u_{m}(z)=z^{m} / \sqrt{m !}\right\}_{|m|=0}^{\infty}$ is an orthonormal family, i.e.,

$$
\left\langle u_{k}, u_{m}\right\rangle_{\mathfrak{F}}=\delta_{k, m}
$$

Moreover, it is complete because in view of (7.2), if $F \in \mathfrak{F}$, then $\sqrt{m !} a_{m}=$ $\left\langle F, u_{m}\right\rangle_{\mathfrak{F}}$ for all multi-indices $m$. Hence, if $\left\langle F, u_{m}\right\rangle_{\mathfrak{F}}=0$ for all $m$, then $F=0$, and it follows that $\left\{u_{m}(z)\right\}_{|m|=0}^{\infty}$ is complete and consequently it is an orthonormal basis of $\mathfrak{F}$.

By applying the Cauchy-Schwarz inequality to the Taylor series of $F=\sum_{m} a_{m} z^{m}$, we obtain

$$
|F(z)|^{2} \leq\left(\sum m !\left|a_{m}\right|^{2}\right)\left(\sum \frac{|z|^{2 m}}{m !}\right)=e^{|z|^{2}}\|F\|_{\mathfrak{F}}^{2},
$$

which shows that convergence in $\mathfrak{F}$ implies pointwise convergence and uniform convergence on compact sets. Another consequence of (7.4) is that the evaluation map $F \rightarrow F(a)$ is continuous; hence, $\mathfrak{F}$ is a reproducing kernel Hilbert space. Since $\left\{u_{m}(z)\right\}_{|m|=0}^{\infty}$ is an orthonormal basis of $\mathfrak{F}$, the reproducing kernel can be found explicitly. In fact, the reproducing kernel is readily seen to be

$$
K(z, w)=\sum_{m} \frac{z^{m} \bar{w}^{m}}{m !}=e^{\langle z, w\rangle} .
$$

Thus, for any $F \in \mathfrak{F}$, we have by (7.2)

$$
\langle F(z), K(z, w)\rangle_{\mathfrak{F}}=\int_{\mathbb{C}^{n}} F(z) e^{\langle w, z\rangle} d \mu(z)=\sum_{n=0}^{\infty} n ! a_{n} \frac{w^{n}}{n !}=F(w) .
$$




\section{THE BARGMANN TRANSFORM}

The Bargmann transform was introduced in [3] to establish the mathematical foundation for some of Foch's work on quantum field theory. It has also appeared in the area of quantum optics 22,23 . Recently, it has appeared in some important applications, one of which is its use in the solution of the Gabor representation problem and in the proof of the completeness of the canonical coherent states in quantum mechanics and the Gabor frames in signal analysis [1,20,21.

Definition 8.1. The Bargmann transform $\mathcal{A}[f]$ of a function $f: \mathbb{R}^{n} \rightarrow \mathbb{C}$ is defined by

$$
\mathcal{A}[f](z)=F(z)=(\pi)^{-n / 4} \int_{\mathbb{R}^{n}} f(q) \exp \left\{-\left(\|z\|^{2}+\|q\|^{2}\right) / 2+\sqrt{2}\langle z, q\rangle\right\} d^{n} q,
$$

which can be written as

$$
F(z)=\langle k(z, q), \bar{f}(q)\rangle_{L^{2}\left(\mathbb{R}^{n}\right)}, \quad \text { whenever } f \in L^{2}\left(\mathbb{R}^{n}\right),
$$

where $z=x+i y$, and

$$
k(z, q)=(\pi)^{-n / 4} \exp \left\{-\left(\|z\|^{2}+\|q\|^{2}\right) / 2+\sqrt{2}\langle z, q\rangle\right\},
$$

$q \in \mathbb{R}^{n}$ and $z \in \mathbb{C}^{n}$.

If $f$ is a locally integrable function such that $f(q)=O\left(\exp \left(a\|q\|^{2}\right)\right)$ for sufficiently large $\|q\|$, and some $a<1 / 2$, in particular, if $f \in L^{2}\left(\mathbb{R}^{n}\right)$, then its Bargmann transform exists almost everywhere.

Let $\tilde{H}_{m}(x)$ be the Hermite polynomial of degree $m$ defined by

$$
\tilde{H}_{m}(x)=(-1)^{m} e^{x^{2}}\left(\frac{d}{d x}\right)^{m} e^{-x^{2}}, 0 \leq m .
$$

We define the normalized Hermite polynomials by

$$
H_{m}(x)=\frac{1}{\sqrt[4]{\pi} 2^{m / 2} \sqrt{m !}} \tilde{H}_{m}(x)
$$

so that

$$
\int_{\mathbb{R}} H_{k}(x) H_{m}(x) e^{-x^{2}} d x=\delta_{k, m}
$$

or

$$
\int_{\mathbb{R}} h_{k}(x) h_{m}(x) d x=\delta_{k, m}
$$

where $h_{k}(x)=H_{k}(x) e^{-x^{2} / 2}$ are the normalized Hermite functions, which are an orthonormal basis of $L^{2}(\mathbb{R})$.

Let $\alpha=\left(\alpha_{1}, \cdots, \alpha_{n}\right)$ be a multi-index with $\alpha_{i}$ being a nonnegative integer and $|\alpha|=\alpha_{1}+\cdots+\alpha_{n}$. We define the $n$-dimensional normalized Hermite polynomial of degree $m$ by

$$
H_{\alpha}(x)=H_{\alpha_{1}}\left(x_{1}\right) \cdots H_{\alpha_{n}}\left(x_{n}\right), \quad|\alpha|=m, x=\left(x_{1}, \cdots, x_{n}\right),
$$

with weight function $W(x)=e^{-\|x\|^{2}}, x \in \mathbb{R}^{n}$. The $n$-dimensional normalized Hermite functions are defined by

$$
h_{\alpha}(x)=H_{\alpha}(x) e^{-\|x\|^{2} / 2},
$$


so that

$$
\int_{\mathbb{R}^{n}} h_{\alpha}(x) h_{\beta}(x) d^{n} x=\delta_{\alpha, \beta}
$$

It then follows from the relation [11, P. 837, Formula 7.374-6]

$$
\int_{\mathbb{R}} e^{-(x-y)^{2}} \tilde{H}_{n}(x) d x=\sqrt{\pi} 2^{n} y^{n}, \quad n=0,1, \ldots,
$$

that the Bargmann transform of the normalized Hermite function $h_{\alpha}(q)$ is $u_{\alpha}(z)=$ $z^{\alpha} / \sqrt{\alpha !},|\alpha|=0,1,2, \ldots$.

Since the Hermite functions $\left\{h_{\alpha}(q)\right\}$ are an orthonormal basis of $L^{2}\left(\mathbb{R}^{n}\right)$, we have for any $f \in L^{2}\left(\mathbb{R}^{n}\right)$,

$$
f(q)=\sum_{\alpha}\left\langle f, h_{\alpha}\right\rangle h_{\alpha}(q) \quad \text { with } \sum_{\alpha}\left|\left\langle f, h_{\alpha}\right\rangle\right|^{2}<\infty .
$$

Therefore, the Bargmann transform, $F(z)=\mathcal{A}[f](z)$, of $f$ is given by

$$
F(z)=\sum_{\alpha}\left\langle f, h_{\alpha}\right\rangle z^{\alpha} / \sqrt{\alpha !}
$$

which is in $\mathfrak{F}$ since by $(7.3)$

$$
\|F\|_{\mathfrak{F}}=\sum_{\alpha}\left|\left\langle f, h_{\alpha}\right\rangle\right|^{2}<\infty .
$$

There are several ways to prove Parseval's relation for the Bargmann transform. The one we shall use is based on formula (7.2). Let $F$ and $G$ be the Bargmann transforms of $f, g \in L^{2}\left(\mathbb{R}^{n}\right)$, respectively. If we denote $\left\langle f, h_{\alpha}\right\rangle$ and $\left\langle g, h_{\alpha}\right\rangle$ by $\hat{f}_{\alpha}$ and $\hat{g}_{\alpha}$, then

$$
\begin{aligned}
\langle f, g\rangle_{L^{2}\left(\mathbb{R}^{n}\right)} & =\int_{\mathbb{R}^{n}}\left(\sum_{|\alpha|=0}^{\infty} \hat{f}_{\alpha} h_{\alpha}(q)\right)\left(\sum_{|\beta|=0}^{\infty} \overline{\hat{g}}_{\beta} \bar{h}_{\beta}(q)\right) d^{n} q \\
& =\sum_{|\alpha|=0}^{\infty} \hat{f}_{\alpha} \overline{\hat{g}}_{\alpha} .
\end{aligned}
$$

On the other hand, we have by Lemma 7.2

$$
\begin{aligned}
\langle F, G\rangle_{\mathfrak{F}} & =\int_{\mathbb{C}^{n}} F(z) \bar{G}(z) d \mu_{n}(z) \\
& =\int_{\mathbb{C}^{n}}\left(\sum_{|\alpha|=0}^{\infty} \hat{f}_{\alpha} u_{\alpha}(z)\right)\left(\sum_{|\beta|=0}^{\infty} \overline{\hat{g}}_{\beta} \bar{u}_{\beta}(z)\right) d \mu_{n}(z) \\
& =\sum_{|\alpha|,|\beta|=0}^{\infty} \hat{f}_{\alpha} \overline{\hat{g}}_{\beta}\left\langle u_{\alpha}, u_{\beta}\right\rangle_{\mathfrak{F}}=\sum_{|\alpha|=0}^{\infty} \hat{f}_{n} \overline{\hat{g}}_{n} .
\end{aligned}
$$

Therefore, we have the following Parseval relation for the Bargmann transform:

$$
\langle F, G\rangle_{\mathfrak{F}}=\langle f, g\rangle_{L^{2}\left(\mathbb{R}^{n}\right)} .
$$

Theorem 8.2. The Bargmann transformation is a unitary transformation from $L^{2}\left(\mathbb{R}^{n}\right)$ onto $\mathfrak{F}$ that maps the normalized Hermite functions $h_{\alpha}(x)$ into $u_{\alpha}(z)=$ $z^{\alpha} / \sqrt{\alpha !},|\alpha|=0,1,2, \ldots$. 


\section{Chromatic expansions in the Bargmann-Segal-Foch space}

In this section we show that functions in the Bargmann-Segal-Foch space can be expanded in chromatic series. To this end we use the Bargmann transform and chromatic derivatives to show that there exists an entire function $\psi$ in the space $\mathfrak{F}$ whose chromatic derivatives $\mathbb{K}^{m}[\psi](z)$ are an orthogonal basis for $\mathfrak{F}$; that is, the basis is generated from one single function by applying successively chromatic differentiations to it.

Let

$$
L_{i}=\frac{1}{\sqrt{2}}\left(\frac{\partial}{\partial z_{i}}+z_{i}\right)
$$

It is easy to see that

$$
L_{i} F(z)=\int_{\mathbb{R}^{n}} f(q) q_{i} k(z, q) d^{n} q
$$

hence

$$
H_{\alpha_{i}}\left(L_{i}\right) F(z)=\int_{\mathbb{R}^{n}} f(q) H_{\alpha_{i}}\left(q_{i}\right) k(z, q) d^{n} q
$$

and consequently

$$
H_{\alpha}(L) F(z)=\int_{\mathbb{R}^{n}} f(q) H_{\alpha}(q) k(z, q) d^{n} q,
$$

where

$$
H_{\alpha}(L)=H_{\alpha_{1}}\left(L_{1}\right) \cdots H_{\alpha_{n}}\left(L_{n}\right) .
$$

Following the results of Section 3 , we have the following definition.

Definition 9.1. We define the $\alpha$-th chromatic derivative of $F(z)$ with respect to the operator $L$ and the Hermite polynomials as

$$
K^{\alpha} F(z)=H_{\alpha}(L) F(z) .
$$

Let $\mathcal{S}\left(\mathbb{R}^{n}\right)$ be the Schwartz space of rapidly decreasing functions consisting of all $\phi \in C^{\infty}\left(\mathbb{R}^{n}\right)$ such that

$$
\gamma_{l, m}(\phi)=\sup _{q \in \mathbb{R}^{n},|\beta| \leq l,|\alpha| \leq m}\left|q^{\beta} \frac{\partial^{|\alpha|} \phi(q)}{\partial q_{1}^{\alpha_{1}} \cdots q_{n}^{\alpha_{n}}}\right|<\infty,
$$

where $|\alpha|=\alpha_{1}+\cdots+\alpha_{n},|\beta|=\beta_{1}+\cdots+\beta_{n}$, and $\alpha_{i}, \beta_{i} \in \mathbb{N}$.

Lemma 9.2. Let $\mathcal{A}(\mathcal{S})=\mathbf{B}$. Then

(i) $\mathbf{B} \subset \mathfrak{F}$, and $\mathbf{B}$ is closed under the chromatic differentiation operator $K^{\alpha}$, but the space $\mathfrak{F}$ is not.

(ii) However, if $F \in \mathfrak{F}$ is such that either $z_{i} F$ or $\frac{\partial F}{\partial z_{i}}$ belongs to $\mathfrak{F}$, so does $L_{i} F$.

(iii) More generally, if $z^{\alpha} F \in \mathfrak{F}$ for all multi-index $\alpha$, then $K^{\alpha} F \in \mathfrak{F}$.

Proof. (i) Clearly $\mathcal{S}\left(\mathbb{R}^{n}\right) \subset L^{2}\left(\mathbb{R}^{n}\right)$, and hence $\mathbf{B} \subset \mathfrak{F}$. Let $\phi \in \mathcal{S}$. Then

$$
\Phi(z)=\mathcal{A}(\phi)(z)=\int_{\mathbb{R}^{n}} \phi(q) k(z, q) d^{n} q,
$$

and hence

$$
\left[K^{\alpha} \Phi\right](z)=\int_{\mathbb{R}^{n}} \phi(q) H_{\alpha}(q) k(z, q) d^{n} q
$$


But

$$
\begin{aligned}
\int_{\mathbb{R}^{n}}\left|\phi(q) H_{\alpha}(q)\right|^{2} d^{n} q & =\int_{\|q\| \leq 1}\left|\phi(q) H_{\alpha}(q)\right|^{2} d^{n} q+\int_{\|q\|>1}\left|\phi(q) H_{\alpha}(q)\right|^{2} d^{n} q \\
& =C+\int_{1<\|q\|}\left|\phi(q) q^{\beta} \frac{H_{\alpha}(q)}{q^{\beta}}\right|^{2} d^{n} q \\
& \leq C+\gamma_{|\beta|, 0}^{2}(\phi) \int_{1<\|q\|}\left|\frac{H_{\alpha}(q)}{q^{\beta}}\right|^{2} d^{n} q .
\end{aligned}
$$

For $\beta$ such that $\alpha_{i}+1<\beta_{i}$ and $i=1,2, \cdots, n$, we obtain that the last integral is convergent and hence $\phi(q) H_{\alpha}(q) \in L^{2}\left(\mathbb{R}^{n}\right)$, and therefore, $\left[K^{\alpha} \Phi\right](z) \in \mathfrak{F}$.

(ii) Let $F(z)=\sum_{|m|=0}^{\infty} a_{m} z^{m}$. Then

$$
z_{i} F(z)=\sum a_{m_{1}, \cdots, m_{i}-1, \cdots, m_{n}} z^{m}
$$

and

$$
\frac{\partial F}{\partial z_{i}}=\sum\left(m_{i}+1\right) a_{m_{1}, \cdots, m_{i}+1, \cdots, m_{n}} z^{m}
$$

From (7.3) we obtain that

$$
\begin{aligned}
\left\|z_{i} F(z)\right\|_{\mathfrak{F}}^{2} & =\sum\left|a_{m_{1}, \cdots, m_{i}-1, \cdots, m_{n}}\right|^{2} m ! \\
& =\sum\left|a_{m}\right|^{2} m_{1} ! \cdots\left(m_{i}+1\right) ! \cdots m_{n} ! \\
& =\sum\left|a_{m}\right|^{2}\left(m_{i}+1\right) m !
\end{aligned}
$$

and similarly

$$
\begin{aligned}
\left\|\frac{\partial F}{\partial z_{i}}\right\|_{\mathfrak{F}}^{2} & =\sum\left|a_{m_{1}, \cdots, m_{i}+1, \cdots, m_{n}}\right|^{2}\left(m_{i}+1\right)^{2} m ! \\
& =\sum\left|a_{m}\right|^{2}\left(m_{i}\right)^{2} m_{1} ! \cdots\left(m_{i}-1\right) ! \cdots m_{n} ! \\
& =\sum\left|a_{m}\right|^{2}\left(m_{i}\right) m ! .
\end{aligned}
$$

From (9.1) and (9.2) it follows that

$$
\left\|z_{i} F(z)\right\|_{\mathfrak{F}}^{2}=\left\|\frac{\partial F}{\partial z_{i}}\right\|_{\mathfrak{F}}^{2}+\|F(z)\|_{\mathfrak{F}}^{2},
$$

which completes the proof of part (ii).

(iii) Finally, for $\beta=\left(\beta_{1}, \cdots, \beta_{n}\right)$ and $\beta_{i} \in \mathbb{N}$ we have

$$
z^{\beta} F(z)=\sum_{m} a_{m} z^{m+\beta},
$$

which in view of (7.3) leads to

$$
\begin{aligned}
\left\|z^{\beta} F(z)\right\|_{\mathfrak{F}}^{2} & =\sum_{m}\left|a_{m}\right|^{2}(m+\beta) ! \\
& =\sum_{m}\left|a_{m}\right|^{2}\left(\prod_{i=1}^{n} Q_{i}\left(m_{i}\right)\right) m !
\end{aligned}
$$

where

$$
Q_{i}\left(m_{i}\right)=\left(m_{i}+\beta_{i}\right)\left(m_{i}+\beta_{i}-1\right) \cdots\left(m_{i}+1\right), i=1,2, \cdots, n,
$$


is a polynomial in $m_{i}$ of degree $\beta_{i}$. Moreover,

$$
\frac{\partial^{|\alpha|} F}{\partial z^{\alpha}}=\sum a_{m}\left(\prod_{i=1}^{n} \frac{m_{i} !}{\left(m_{i}-\alpha_{i}\right) !}\right) z^{m-\alpha} .
$$

Hence,

$$
z^{\alpha} \frac{\partial^{|\alpha|} F}{\partial z^{\alpha}}=\sum a_{m}\left(\prod_{i=1}^{n} \frac{m_{i} !}{\left(m_{i}-\alpha_{i}\right) !}\right) z^{m}
$$

and it follows that

$$
\left\|z^{\alpha} \frac{\partial^{|\alpha|} F}{\partial z^{\alpha}}\right\|_{\mathfrak{F}}^{2}=\sum\left|a_{m}\right|^{2}\left(\prod_{i=1}^{n} P_{i}\left(m_{i}\right)\right)^{2} m !
$$

where

$$
P_{i}\left(m_{i}\right)=\frac{m_{i} !}{\left(m_{i}-\alpha_{i}\right) !}, \quad i=1, \cdots, n,
$$

is a polynomial of degree $\alpha_{i}$ in $m_{i}$.

By combining (9.3) and (9.4), we obtain that for $\beta$ such that $2 \alpha_{i}<\beta_{i}$ and all $i$,

$$
\left\|z^{\alpha} \frac{\partial^{|\alpha|} F}{\partial z^{\alpha}}\right\|_{\mathfrak{F}}^{2} \leq\left\|z^{\beta} F(z)\right\|_{\mathfrak{F}}^{2} .
$$

Similarly, one can show that

$$
\left\|z^{\delta} \frac{\partial^{|\alpha|} F}{\partial z^{\alpha}}\right\|_{\mathfrak{F}}^{2} \leq\left\|z^{\alpha} \frac{\partial^{|\alpha|} F}{\partial z^{\alpha}}\right\|_{\mathfrak{F}}^{2} \leq\left\|z^{\beta} F(z)\right\|_{\mathfrak{F}}^{2}, \quad \text { if } \delta_{i} \leq \alpha_{i} \leq \beta_{i} / 2 .
$$

Since $K^{\alpha} F(z)=H_{\alpha_{1}}\left(L_{1}\right) \cdots H_{\alpha_{n}}\left(L_{n}\right) F(z)$ is a product of $n$ polynomials in which the $i$-th polynomial is of degree $\alpha_{i}$ in $L_{i}$, it is easy to see that the general term of this polynomial is of the form

$$
z_{i}^{\delta_{i}} \frac{\partial^{\gamma_{i}} F}{\partial z_{i}^{\gamma_{i}}}, \quad \text { where } 0 \leq \delta_{i}+\gamma_{i} \leq \alpha_{i}
$$

and hence the general term in $K^{\alpha} F(z)$ is of the form

$$
z^{\delta} \frac{\partial^{|\gamma|} F}{\partial z^{\gamma}}, \quad \text { where }|\delta|+|\gamma| \leq|\alpha| \text {. }
$$

Thus, from (9.5) it now follows that if $z^{\beta} F \in \mathfrak{F}$ for all $\beta$, then $K^{\alpha} F \in \mathfrak{F}$.

Finally, we are ready to derive the main result of this section.

Theorem 9.3. There exists a function $\phi \in \mathcal{S}\left(\mathbb{R}^{n}\right)$ whose Bergmann transform $\mathcal{A}(\phi)=\psi(z) \in \mathfrak{F}$ has the property that its chromatic derivatives $\left\{K^{\alpha} \psi(z)\right\}$ are an orthogonal basis of $\mathfrak{F}$. Hence, any $F \in \mathfrak{F}$ can be written in the form

$$
F(z)=\sum_{\alpha} K^{\alpha} F(0) K^{\alpha} \psi(z)
$$

Proof. Let $F(z)$ be the Bergmann transform of $f \in L^{2}\left(\mathbb{R}^{n}\right)$. Then

$$
\begin{aligned}
K^{\alpha} F(0) & =H_{\alpha}(L) F(0)=c^{\prime} \int_{\mathbb{R}^{n}} f(q) H_{\alpha}(q) \exp \left(-\|q\|^{2} / 2\right) d^{n} q \\
& =c^{\prime} \int_{\mathbb{R}^{n}} f(q) h_{\alpha}(q) d^{n} q=c^{\prime}\left\langle f, h_{\alpha}\right\rangle=c^{\prime} \hat{f}_{\alpha},
\end{aligned}
$$


where $c^{\prime}=(\pi)^{-n / 4}$. For $f \in L^{2}\left(\mathbb{R}^{n}\right)$, we have $f=\sum_{\alpha}\left\langle f, h_{\alpha}\right\rangle h_{\alpha}$. Thus

$$
\begin{aligned}
F(z) & =\sum_{\alpha}\left\langle f, h_{\alpha}\right\rangle \int_{\mathbb{R}^{n}} h_{\alpha}(q) k(z, q) d^{n} q \\
& =\left(1 / c^{\prime}\right) \sum_{\alpha} K^{\alpha} F(0) \int_{\mathbb{R}^{n}} h_{\alpha}(q) k(z, q) d^{n} q .
\end{aligned}
$$

Now let $\phi(q)=\left(1 / c^{\prime}\right) \exp \left(-\|q\|^{2} / 2\right)$. Then

$$
\begin{aligned}
\psi(z) & =\left(1 / c^{\prime}\right) \int_{\mathbb{R}^{n}} \exp \left(-\|q\|^{2} / 2\right) k(z, q) d^{n} q \\
& =\int_{\mathbb{R}^{n}} \exp \left[-\|q\|^{2} / 2-\left(\|q\|^{2}+\|z\|^{2}\right) / 2+\sqrt{2}\langle z, q\rangle\right] d^{n} q \\
& =\prod_{i=1}^{n}\left(\int_{\mathbb{R}} \exp \left(-q_{i}^{2}-z_{i}^{2} / 2+\sqrt{2} z_{i} q_{i}\right) d q_{i}\right) \\
& =\prod_{i=1}^{n}\left(\int_{\mathbb{R}} \exp \left[-\left(q_{i}-z_{i} / \sqrt{2}\right)^{2}\right] d q_{i}\right)=\pi^{n / 2} .
\end{aligned}
$$

Since ([11, P. 338, Formula 3.462-6])

$$
\int_{\mathbb{R}} x \exp \left\{-(x-b)^{2}\right\} d x=\sqrt{\pi} b
$$

we have

$$
\begin{aligned}
L_{j} \psi(z) & =\left(\int_{\mathbb{R}} q_{j} \exp \left[-\left(q_{j}-z_{j} / \sqrt{2}\right)^{2}\right] d q_{j}\right) \\
& \times \prod_{i=1, i \neq j}^{n}\left(\int_{\mathbb{R}} \exp \left[-\left(q_{i}-z_{i} / \sqrt{2}\right)^{2}\right] d q_{i}\right) \\
& =\frac{\pi^{n / 2}}{\sqrt{2}} z_{j} .
\end{aligned}
$$

By applying the operator $K^{\alpha}$ to $\psi$, we obtain

$$
\begin{aligned}
K^{\alpha} \psi(z) & =H_{\alpha}(L) \psi(z) \\
& =\int_{\mathbb{R}^{n}} H_{\alpha}(q) \exp \left[-\sum_{i=1}^{n}\left(q_{i}-z_{i} / \sqrt{2}\right)^{2}\right] d^{n} q \\
& =\int_{\mathbb{R}^{n}} h_{\alpha}(q) \exp \left[-\sum_{i=1}^{n}\left(q_{i}^{2}+z_{i}^{2}\right) / 2+\sqrt{2} \sum_{i=1}^{n} z_{i} q_{i}\right] d^{n} q \\
& =\left(1 / c^{\prime}\right) \int_{\mathbb{R}^{n}} h_{\alpha}(q) k(z, q) d^{n} q .
\end{aligned}
$$

Therefore, by substituting (9.12) into (9.8), we obtain (9.6).

To complete the proof, we need to show that $\left\{K^{\alpha} \psi(z)\right\}_{\alpha}$ is an orthogonal set. From (9.12), we have

$$
K^{\alpha} \psi(z)=\int_{\mathbb{R}^{n}} H_{\alpha}(q) \exp \left[-\sum_{i=1}^{n}\left(q_{i}-z_{i} / \sqrt{2}\right)^{2}\right] d^{n} q .
$$


But from (8.3), we have for $m \in \mathbb{N}$

$$
\begin{aligned}
\int_{\mathbb{R}} H_{m}(x) & \exp \left[-(x-z / \sqrt{2})^{2}\right] d x \\
& =\frac{1}{\sqrt[4]{\pi} 2^{m / 2} \sqrt{m !}} \int_{\mathbb{R}} \tilde{H}_{m}(x) \exp \left[-(x-z / \sqrt{2})^{2}\right] d x \\
& =\frac{1}{\sqrt[4]{\pi} 2^{m / 2} \sqrt{m !}} \sqrt{\pi} 2^{m}\left(\frac{z}{\sqrt{2}}\right)^{m}=\frac{\sqrt[4]{\pi}}{\sqrt{m !}} z^{m} .
\end{aligned}
$$

Hence, it follows that

$$
\begin{aligned}
K^{\alpha} \psi(z) & =\frac{(\sqrt[4]{\pi})^{n}}{\sqrt{\alpha_{1} ! \cdots \alpha_{n} !}} z_{1}^{\alpha_{1}} \cdots z_{n}^{\alpha_{n}} \\
& =\frac{(\sqrt[4]{\pi})^{n}}{\sqrt{\alpha !}} z^{\alpha} .
\end{aligned}
$$

The proof is now complete in view of Lemma 7.2

\section{Concluding Remarks}

As we have pointed out in the Introduction, unlike other notions of classical derivatives, such as symmetric, Peano, $L^{p}$, quantum, Fréchet, and Gateaux derivatives, which are generalizations of the ordinary derivatives that are used to describe the local behavior of a function in a neighborhood of the point of differentiation, chromatic derivatives arose from real world applications in signal processing and can be used to describe the global behavior of analytic functions as we have demonstrated in Sections 4 and 9 .

One of the goals of this article is to draw the attention of the mathematics community to this novel notion of derivatives. But as the anonymous referee has remarked, whether chromatic derivatives will prove to be useful in mathematical applications is to be seen. In this concluding section we point out two potential applications of chromatic derivatives. The first is in asymptotic expansions and the second is in differential equations.

In 25, 26 the authors investigated the asymptotic behavior for the large argument $t$ of the Cesàro means of the Fourier transform of polynomials $\left\{P_{n}(x)\right\}$ orthogonal with respect to a measure $d \mu$ on the real line. More precisely, let $d \mu$ be a positive Borel measure supported on the real axis for which the moment problem is determined, i.e., all the moments $\mu_{n}=\int x^{n} d \mu(x)$ exist and are finite. This includes measures with unbounded support and compactly supported multi-fractals measures. Let $\left\{P_{n}(x)\right\}$ be the orthonormal polynomials associated with $d \mu$, so that

$$
\int P_{n}(x) P_{m}(x) d \mu(x)=\delta_{m, n}
$$

Consider what the authors call the generalized Fourier-Bessel functions (F-B functions for short):

$$
\mathfrak{F}_{n}(\mu ; t)=\int P_{n}(x) e^{-i t x} d \mu(x) .
$$

The focus of [25] was to study the long term behavior of the Cesàro averages

$$
\overline{\mathfrak{F}}_{n}(\mu ; t)=C\left(\mathfrak{F}_{n} ; t\right)=\frac{1}{2 t} \int_{-t}^{t} \mathfrak{F}_{n}(\mu ; x) d \mu(x)=\int P_{n}(x) \operatorname{Sinc}(t x) d \mu(x)
$$


and the quadratic amplitudes $A_{m, n}(\mu ; t)$ defined by

$$
A_{m, n}(\mu ; t)=C\left(\mathfrak{F}_{m} \mathfrak{F}_{n}^{*} ; t\right)=\frac{1}{2 t} \int_{-t}^{t} \mathfrak{F}_{m}(\mu ; x) \mathfrak{F}_{n}^{*}(\mu ; x) d \mu(x)
$$

In the language of chromatic derivatives, $\mathfrak{F}_{n}$ is the $n$-th chromatic derivative of the Fourier transform of the measure $\mu$ with respect to the orthogonal polynomials $\left\{P_{n}\right\}$ and the operator $i d / d t$ as can be seen from the equation

$$
K^{n}(\hat{\mu})(t)=P_{n}\left(i \frac{d \hat{\mu}}{d t}\right)(t)=\int P_{n}(x) e^{-i t x} d \mu(x) .
$$

Hence, $A_{m, n}$ is the Cesàro average of the product of two chromatic derivatives. For the relationship between $\mathfrak{F}_{n}(\mu ; t)$ and the quantum time evolution, and their asymptotic behavior, see [25, 26].

Equation (10.1) leads to the following problem: Given $f$ and an orthogonal polynomial $\left\{P_{n}(x)\right\}$, find $y$ so that

$$
\int P_{n}(x) e^{-i t x} y(x) d x=f(t) .
$$

This last equation can be viewed as a simple chromatic differential equation in which

$$
K^{n}[\hat{y}](t)=f(t),
$$

where the chromatic derivative is taken with respect to the differential operator $i d / d t$.

In what follows, we shall briefly outline how to solve more general chromatic differential equations with constant coefficients. Consider the chromatic differential equation

$$
\mathcal{L} y=\sum_{k=0}^{n} a_{k} K^{k}[y](x)=f(x),
$$

where $a_{k}$ are constants, $f$ is a known function, and the chromatic derivatives are taken with respect to a differential operator $L$ as given in Section 3.2, and a system of orthonormal polynomials $\left\{P_{n}\right\}$ on $I$. Let $\phi(x, \lambda)$ have the same meaning as in Section 3.2. and for the sake of simplicity, let us further assume that $L$ is self-adjoint. Consider the integral transform

$$
\mathcal{F}(g)(\lambda)=G(\lambda)=\int_{I} g(x) \phi(x, \lambda) d x
$$

whose inverse is assumed to be of the form

$$
\mathcal{F}^{-1}(G)(x)=g(x)=\int G(\lambda) \psi(x, \lambda) d \lambda
$$

for some kernel function $\psi(x, \lambda)$. In some cases of interest, $\psi=\phi^{*}$ as in the Fourier transform or $\psi=\phi$ as in the self-symmetric transforms, such as the Hankel transform.

Multiplying (10.2) by $\phi$ and integrating over $I$, we obtain

$$
\langle\mathcal{L} y, \phi\rangle=\langle f, \phi\rangle \text {. }
$$


Thus,

$$
\begin{aligned}
\langle\mathcal{L} y, \phi\rangle & =\left\langle\sum_{k=0}^{n} a_{k} K^{k}[y](x), \phi\right\rangle=\sum_{k=0}^{n} a_{k}\left\langle y(x), K^{k}[\phi]\right\rangle \\
& =\sum_{k=0}^{n} a_{k}\left\langle y(x), P_{k}(L) \phi\right\rangle=\sum_{k=0}^{n} a_{k}\left\langle y(x), P_{k}(\lambda) \phi\right\rangle \\
& =\sum_{k=0}^{n} a_{k} P_{k}(\lambda)\langle y(x), \phi\rangle=\sum_{k=0}^{n} a_{k} P_{k}(\lambda) Y(\lambda)=F(\lambda),
\end{aligned}
$$

where

$$
Y(\lambda)=\langle y(x), \phi\rangle=\int_{I} y(x) \phi(x, \lambda) d x \quad \text { and } F(\lambda)=\int_{I} f(x) \phi(x, \lambda) d x .
$$

From (10.4), we obtain

$$
Y(\lambda)=\frac{F(\lambda)}{\sum_{k=0}^{n} a_{k} P_{k}(\lambda)}
$$

from which it follows that

$$
y(x)=\mathcal{F}^{-1}\left(\frac{F(\lambda)}{\sum_{k=0}^{n} a_{k} P_{k}(\lambda)}\right)=\int \frac{F(\lambda)}{\sum_{k=0}^{n} a_{k} P_{k}(\lambda)} \psi(x, \lambda) d \lambda .
$$

In closing, we have shown that chromatic derivatives have more practical and mathematical applications than other notions of classical derivatives. Therefore, we hope that some readers may find them interesting and discover new uses for them. The extension of some of the ideas presented in this article on Hilbert and Banach spaces will be the subject of future research.

\section{REFERENCES}

[1] V. Bargmann, P. Butera, L. Girardello, and John R. Klauder, On the completeness of the coherent states, Rep. Mathematical Phys. 2 (1971), no. 4, 221-228. MR.0290680 (44 \#7860)

[2] V. Bargmann, On a Hilbert space of analytic functions and an associated integral transform. Part II. A family of related function spaces. Application to distribution theory, Comm. Pure Appl. Math. 20 (1967), 1-101. MR0201959 (34 \#1836)

[3] V. Bargmann, On a Hilbert space of analytic functions and an associated integral transform, Comm. Pure Appl. Math. 14 (1961), 187-214. MR0157250 (28 \#486)

[4] J. Byrnes, Local signal reconstruction via chromatic differentiation filter banks, Conference Record of the Thirty-Fifth Asilomar conference on Signals, Systems and Computers 2001, Vol. 1 (2001), 568-572.

[5] M. Cushman and T. Herron, "The general theory of chromatic derivatives", Kromos Technology Technical Report (2001).

[6] M. Cushman, M. Narasimha, and P.P. Vaidyanathan, Finite-channel chromatic derivative filter banks, IEEE Signal Processing Letters, Vol. 10 (1), (2003), 15-17.

[7] Charles F. Dunkl and Yuan Xu, Orthogonal polynomials of several variables, Encyclopedia of Mathematics and its Applications, vol. 81, Cambridge University Press, Cambridge, 2001. MR:1827871 (2002m:33001)

[8] Peter Duren and Alexander Schuster, Bergman spaces, Mathematical Surveys and Monographs, vol. 100, American Mathematical Society, Providence, RI, 2004. MR2033762 (2005c:30053)

[9] T. Herron and J. Byrnes, "Families of orthogonal differential operators for signal procesing", Kromos Technology Technical Report (2001).

[10] V. Foch, Verallgemeinerung und Lösung der Diracschen statistischen Gleichung, Z. Phys., Vol. 49 (1928), pp. $339-357$.

[11] I. Gradshteyn and I. Ryzhik, Tables of Integrals, Series, and Products, Academic Press, New York (1965). 
[12] Aleksandar Ignjatovic and Ahmed I. Zayed, Multidimensional chromatic derivatives and series expansions, Proc. Amer. Math. Soc. 139 (2011), no. 10, 3513-3525, DOI 10.1090/S00029939-2011-10789-5. MR 2813383 (2012f:41042)

[13] A. Ignjatovic, Frequency estimation using time domain methods based on robust differential operators, 2010 IEEE 10th International Conference on Signal Processing (ICSP) (2010) 151-154.

[14] Aleksandar Ignjatović, Chromatic derivatives, chromatic expansions and associated spaces, East J. Approx. 15 (2009), no. 3, 263-302. MR.2741822(2011j:94054)

[15] Aleksandar Ignjatović, Local approximations based on orthogonal differential operators, J. Fourier Anal. Appl. 13 (2007), no. 3, 309-330, DOI 10.1007/s00041-006-6085-y. MR2334612 (2008i:41038)

[16] A. Ignjatovic, Numerical differentiation and signal processing, Proc. Intenational Conference Information, Communications and Signal Processing (ICICS), Singapore (2001).

[17] A. Ignjatovic, Local approximations and signal processing , Kromos Technology Technical Report, Los Altos (2001).

[18] A. Ignjatovic, Numerical differentiation and signal processing, Kromos Technology Technical Report, Los Altos (2001).

[19] A. Ignjatovic and N. Carlin, Signal processing with local behavior, Provisional Patent Application, (60)-143,074 (1999), US Patent Office, Patent issued as US 6313778, June 2001.

[20] A. J. E. M. Janssen, Bargmann transform, Zak transform, and coherent states, J. Math. Phys. 23 (1982), no. 5, 720-731, DOI 10.1063/1.525426. MR655886 (84h:81041)

[21] A. J. E. M. Janssen, Gabor representation of generalized functions, J. Math. Anal. Appl. 83 (1981), no. 2, 377-394, DOI 10.1016/0022-247X(81)90130-X. MR641340(83h:94005)

[22] J. Klauder and B. S. Skagerstam, Coherent States, World Scientific, Singapore (1985). MR.826247(87a:81070)

[23] John R. Klauder and E. C. G. Sudarshan, Fundamentals of quantum optics, W. A. Benjamin, Inc., New York-Amsterdam, 1968. MR0231591 (37 \#7144)

[24] N. N. Lebedev, Special functions and their applications, Dover Publications Inc., New York, 1972. Revised edition, translated from the Russian and edited by Richard A. Silverman; unabridged and corrected republication. MR0350075 (50 \#2568)

[25] Giorgio Mantica and Sandro Vaienti, The asymptotic behaviour of the Fourier transforms of orthogonal polynomials. I. Mellin transform techniques, Ann. Henri Poincaré 8 (2007), no. 2, 265-300, DOI 10.1007/s00023-006-0308-2. MR2314448 (2008m:42043)

[26] Giorgio Mantica and Davide Guzzetti, The asymptotic behaviour of the Fourier transforms of orthogonal polynomials. II. L.I.F.S. measures and quantum mechanics, Ann. Henri Poincaré 8 (2007), no. 2, 301-336, DOI 10.1007/s00023-006-0309-1. MR2314450(2008m:42044)

[27] Vladimir A. Marchenko, Sturm-Liouville operators and applications, Operator Theory: Advances and Applications, vol. 22, Birkhäuser Verlag, Basel, 1986. Translated from the Russian by A. Iacob. MR897106 (88f:34034)

[28] M.A. Naimark, Linear Differential Operators I, George Harrap, London, 1967.

[29] M. Narasimha, A. Ignjatovic, and P. Vaidyanathan, Chromatic derivative filter banks, IEEE Signal Processing Lett., Vol. 9 (7), (2002), pp. 215-216.

[30] B. Savkovic, Decorrelation properties of chromatic derivative signal representation, IEEE Signal Processin Letters, Vol. 17 (8), (2010), pp. 770-773.

[31] G. Szegö, Orthogonal Polynomials, Amer. Math. Soc., Providence, RI (1975).

[32] E. C. Titchmarsh, Eigenfunction expansions associated with second-order differential equations. Part I, Second Edition, Clarendon Press, Oxford, 1962. MR0176151 (31 \#426)

[33] P. Vaidyanathan, A. Ignjatovic, and M. Narasimha, New sampling expansions of bandlimited signals based on chromatic derivatives, Proc. 35th Asilomar Conf. Signals, Systems and Computers, Monterey (2001), pp. 558-562.

[34] Gilbert G. Walter and Xiaoping Shen, A sampling expansion for nonbandlimited signals in chromatic derivatives, IEEE Trans. Signal Process. 53 (2005), no. 4, 1291-1298, DOI 10.1109/TSP.2005.843745. MR2128248 (2005j:94012)

[35] Gilbert G. Walter, Chromatic series with prolate spheroidal wave functions, J. Integral Equations Appl. 20 (2008), no. 2, 263-280, DOI 10.1216/JIE-2008-20-2-263. MR2418070 (2010b:41025) 
[36] Ahmed I. Zayed, Chromatic expansions of generalized functions, Integral Transforms Spec. Funct. 22 (2011), no. 4-5, 383-390, DOI 10.1080/10652469.2010.541059. MR2801291 (2012i:44006)

[37] Ahmed I. Zayed, Generalizations of chromatic derivatives and series expansions, IEEE Trans. Signal Process. 58 (2010), no. 3, 1638-1647, DOI 10.1109/TSP.2009.2038415. MR.2730105 (2011f:94098)

[38] Ahmed I. Zayed, Advances in Shannon's sampling theory, CRC Press, Boca Raton, FL, 1993. MR.1270907 (95f:94008)

Department of Mathematical Sciences, DePaul University, Chicago, Illinois 60614

E-mail address: azayed@condor.depaul.edu 